\title{
Review \\ Graph-Based Conversational AI: Towards a Distributed and Collaborative Multi-Chatbot Approach for Museums
}

\author{
Savvas Varitimiadis ${ }^{1,2}$, Konstantinos Kotis ${ }^{1, * \mathbb{D}}$, Dimitra Pittou ${ }^{2}$ and Georgios Konstantakis ${ }^{2}$ \\ 1 I-Lab, Department of Cultural Technology and Communication, University of the Aegean, \\ 81100 Mytilene, Greece; svaritimiadis@aegean.gr \\ 2 Aegean Solutions S.A., 71202 Heraklion, Greece; pittou@aegeansolutions.com (D.P.); \\ gkonstantakis@aegeansolutions.com (G.K.) \\ * Correspondence: kotis@aegean.gr; Tel.: +30-22510-36620
}

Citation: Varitimiadis, S.; Kotis, K. Pittou, D.; Konstantakis, G. GraphBased Conversational AI: Towards a Distributed and Collaborative MultiChatbot Approach for Museums. Appl. Sci. 2021, 11, 9160. https:// doi.org/10.3390/app11199160

Academic Editor: Tobias Meisen

Received: 31 August 2021

Accepted: 29 September 2021

Published: 1 October 2021

Publisher's Note: MDPI stays neutral with regard to jurisdictional claims in published maps and institutional affiliations.

Copyright: (c) 2021 by the authors. Licensee MDPI, Basel, Switzerland. This article is an open access article distributed under the terms and conditions of the Creative Commons Attribution (CC BY) license (https:// creativecommons.org/licenses/by/ $4.0 /)$.

\begin{abstract}
Nowadays, museums are developing chatbots to assist their visitors and to provide an enhanced visiting experience. Most of these chatbots do not provide a human-like conversation and fail to deliver the complete requested knowledge by the visitors. There are plenty of stand-alone museum chatbots, developed using a chatbot platform, that provide predefined dialog routes. However, as chatbot platforms are evolving and AI technologies mature, new architectural approaches arise. Museums are already designing chatbots that are trained using machine learning techniques or chatbots connected to knowledge graphs, delivering more intelligent chatbots. This paper is surveying a representative set of developed museum chatbots and platforms for implementing them. More importantly, this paper presents the result of a systematic evaluation approach for evaluating both chatbots and platforms. Furthermore, the paper is introducing a novel approach in developing intelligent chatbots for museums. This approach emphasizes graph-based, distributed, and collaborative multi-chatbot conversational AI systems for museums. The paper accentuates the use of knowledge graphs as the key technology for potentially providing unlimited knowledge to chatbot users, satisfying conversational AI's need for rich machine-understandable content. In addition, the proposed architecture is designed to deliver an efficient deployment solution where knowledge can be distributed (distributed knowledge graphs) and shared among different chatbots that collaborate when is needed.
\end{abstract}

Keywords: museum chatbot; conversational AI; knowledge graphs; distributed AI; collaborative AI; multi-agent systems

\section{Introduction}

Conversational AI concerns the study of techniques for software agents that can be engaged in natural conversations with humans or other agents. This includes software such as voice assistants and chatbots. The first generation of this software focused on short task-oriented dialogs [1] such as music playback (e.g., "Alexa, play music") or information retrieval (e.g., "Cortana, what is the weather today in Athens?"). The current challenge in this area is a constant, coherent, and appealing dialogue, as current software is far from being able to have natural everyday conversations with humans [2]. Chatbots are such intelligent software systems that can chat online with users by sending automated messages in text or voice. They can imitate human behavior, talk to people and to other chatbots [3]. The chat approach is designed in such way as to give human-users the impression that they are chatting with another human.

Designing and creating an AI chatbot for a museum is a highly challenging process, as many different parameters must be taken into consideration. Chatbot developers in collaboration with museum stakeholders must identify the characteristics and the needs of the museum and the scope of the proposed chatbot solution. Visitors' engagement and attraction, size and location of the museum, content presentation and manipulation, and 
financial capacities are some crucial factors that determine the type and the capabilities of the proposed AI chatbot solution. The designers must also design on a long-term basis and consider factors such as sustainability, interoperability, scalability, usability, and others [4-9].

Most of the presented museum chatbots rely on ready-made conversational routes providing limited and predefined domain knowledge about the museum and its exhibits. These chatbots are developed with well-known chatbot platforms such as Wit.ai, Google's Dialogflow, IBM's Watson, and others. Additionally, usually lack the ability to conduct a fully successful human-like conversation, and sometimes fail to answer even simple informative questions (if the relative knowledge is missing from their knowledge base). Users do not have the opportunity to place their own custom (free) questions, and when they do, they usually receive an answer of ignorance or/and guidance to other topics.

Intelligent museum chatbots are designed to conduct simple dialogues by exploiting Artificial Intelligence (AI) techniques such as ones based on Machine Learning (ML) and Natural Language Understanding (NLU). ML focuses on engineering chatbots to "learn" how to respond to human-made questions by utilizing supervised or unsupervised algorithms [10,11], while NLU focuses on techniques that allow chatbots to segment, assign meaning, analyze human language in its natural forms and transform it to machine language $[12,13]$. The design and creation of a chatbot with strong conversational skills requires massive and long-term training with big data, along with huge computational and/or manual effort.

The main limitations in current museum chatbot applications are (a) that the designed conversations are not human-like, lacking meaningful interaction and engagement, and (b) that limited knowledge is provided in response to user's questions [6-9]. Therefore, during the design of a museum chatbot all stakeholders must focus on the following key questions:

1. How will our chatbot be able to conduct a human-like conversation?

2. Does our chatbot have access to all the necessary knowledge in order to be able to provide the right answer in a comprehensive manner?

3. How can this be done more effectively with low computational and/or manual effort?

This paper surveys current trends in museum AI chatbots and conversational AI platforms in general, motivated by the real need to introduce and utilize a new architecture that will deliver a museum conversational AI system capable of conducting human-like conversations, utilizing knowledge without limits. This need has been identified in the context of a research and development project for the Nikos Kazantzakis Museum in Greece. The proposed architecture aims to answer the abovementioned key questions by focusing to the design of a system that utilizes Knowledge Graphs (KG), has strong conversational skills by exploiting both NLU and ML techniques, and rely on the distributed and collaborative aspects of AI.

A KG mainly describes real-world entities and their interrelations, organized in a large, directed graph (both a schema and the corresponded data are represented in the graph, lately with the widely used and well-known RDF model, in the form of triplelike statements). The integration of KGs to conversational AI (usually called hybrid AI) provides better rational dialogues that could eventually lead to more meaningful AI chatbot applications [14,15]. In Figure 1 an exemplary KG about the Greek writer Nikos Kazantzakis and related entities is depicted. 


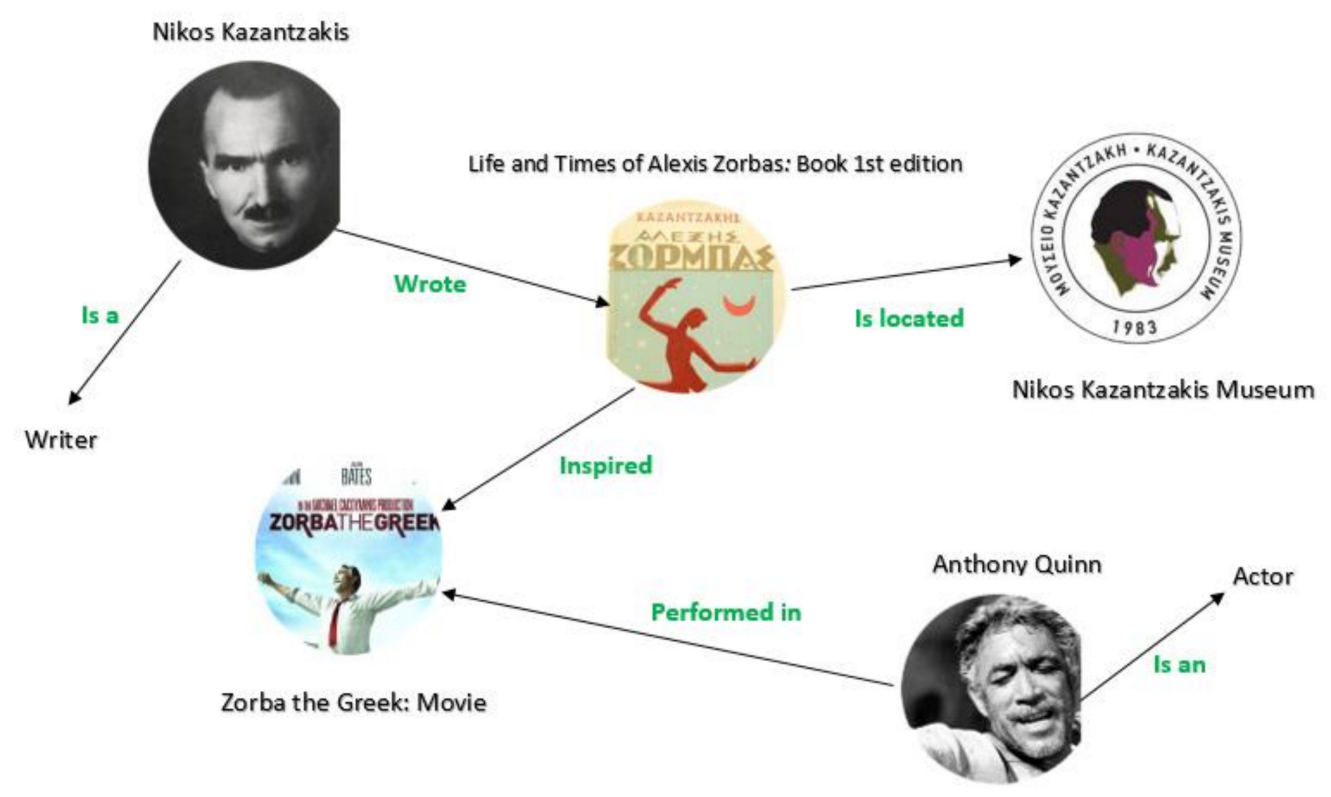

Figure 1. An exemplary knowledge graph representing knowledge about the Greek writer Nikos Kazantzakis and related entities, e.g., museums, actors, movies.

In our early research [16] we have demonstrated preliminary results related to such an approach. Specifically, we introduced a single-chatbot system architecture that aims to retrieve knowledge from various KGs. A preliminary evaluation of a proof-of-concept system concerning the flow of the information and the interaction between the chatbot, and the KGs has been presented. In the current paper, we built upon the single-chatbot architecture, and we extend it by placing it within a novel approach of a knowledge graph-based distributed and collaborative system architecture, where several distributed AI chatbots, "carrying" their own distributed knowledge (encoded in their own KG and partially in the KGs of others), can "work" individually or in collaboration with others (in teams) at the same time. Such an approach aims to design museum chatbots that can provide robust and potentially unlimited knowledge to their visitors about the exhibits and the museum thematics, or even about knowledge that is far from being pre-engineered in the museum's knowledge base (e.g., retrieved from the Semantic Web). In such a multichatbot approach, the collaborating chatbots do not rely on a single central knowledge repository. They work in a distributed manner, utilizing different KGs (e.g., for different museum exhibitions and departments), providing this way more accurate and immediate answers to visitors' questions. In addition, these chatbots can collaborate and help each other by sharing knowledge and experiences that others do not have at the time of its initial acquisition.

The contribution per section and the structure of this survey paper is as follows: Section 2 presents the research methodology of this survey paper. Section 3 presents a short analysis of background knowledge and main concepts used in the paper. Section 4 presents a representative set of developed museum chatbots and their evaluation focusing on the use of KGs and on human-like conversational features. Section 5 presents an evaluation of selected chatbot platforms, and their evaluation conducted with the use of the AHP evaluation method. Section 6 presents a novel architecture of a distributed, collaborative, graph-based conversational AI multi-chatbot system approach. Finally, Section 7 presents the first steps towards an implementation of the proposed architecture for Nikos Kazantzakis Museum in Crete. Section 8 concludes the paper.

\section{Research Methodology}

The research methodology followed in this paper focuses mainly on the collection of information sources related to museum chatbots and AI chatbot platforms that were used 
to develop them. The research conducted in a period of six months, examining academic articles, official webpages, and other relevant web resources. Especially for the museum chatbots, research on academic web portals such as Academia [17], ResearchGate [18], Google [19], and Semantic Scholar [20], was conducted, as well as on online accessible journals of ACM [21], IEEE [22], and Springer [23]. The specific search terms used in various combinations were: "chatbot", "museum" "conversational AI", "artificial intelligence", "cultural organization", "intelligent system". The papers that were collected were mainly related to the domains of museum studies, cultural computing, and computing.

The Web search results included: (a) webpages that were presenting specific museum chatbots, and (b) webpages that were briefly presenting a selected set of museum chatbots $[24,25]$. Furthermore, search on related academic articles, official webpages of the chatbots and other related web sources was conducted. From these search results, all articles that were outdated were excluded, as the referenced chatbots were not using recent AI technologies such as ML, NLP, and KGs. In addition, results related to chatbots that were not developed with a chatbot platform, but with a custom programming language, were excluded. As a further step in research methodology, a more detailed search was conducted by combining search terms with the terms: "Machine Learning", 'ML', 'NLP', 'KGs', 'Natural Language Processing', 'Knowledge Graphs'. With this search more specific articles and Web sources were collected.

Regarding chatbot platforms research, the focus was on platforms that were used in the development of museum chatbots. The official Web resources of the platforms were studied, and related open access articles were retrieved. The research was mainly focused on articles where the developed chatbots used AI technologies, and especially KGs. In addition, research on articles that are evaluating chatbot platforms was conducted, and finally, more specific research was conducted related to how and if the researched platforms support a distributed, collaborative, and multi-chatbot approach.

The research methodology described in this section has been conducted with resources (webpages, articles, books) published in the past 5 years.

\section{Background Knowledge and Preliminaries}

In this section, background knowledge and preliminaries that are necessary for understanding the concept of a KG-based, distributed, and collaborative multi-chatbot system are presented. Starting from the definition of a chatbot (or chatterbot, bot, smart bot, conversational interface, or artificial conversational entity), it is "a computer program or an artificial intelligence which conducts a conversation via auditory or textual methods". A key feature of a chatbot is its ability to serve its users anytime, 24/7, and if it gets intelligent enough, it can provide a satisfactory human-like assistance [4,26-28]. Chatbot applications are currently using the most advanced AI technologies, as presented in the following subsections.

\subsection{Machine Learning}

Machine Learning (ML) is the field of AI science that focuses on getting machines to "learn" and to continually develop autonomously. ML utilizes supervised or unsupervised algorithms such as decision trees, neural networks, deep learning, and others that enable computer systems to optimize the ways of problem solving $[10,11,29,30]$. For AI chatbots, ML is the technology that assist software agents to learn how human agents interact, to train AI models with dialogue examples, to analyze and understand user intent in real-time.

\subsection{Natural Language Understanding}

Natural Language Understanding (NLU) is one of the most important components of an AI chatbot system, as language is an evolving entity with different levels of complexity. NLU provides chatbots the ability to understand and interpret the semantics of natural language and derive the real meaning by exploiting various techniques like name entity recognition, relationship extraction, sentiment analysis, speech recognition and others [12]. 
Natural Language Processing (NLP) is part of an NLU strategy which allows computers to segment, assign meaning, and analyze human communication in its natural forms. NLP utilizes several AI techniques such as language parsing, part-of-speech (POS) tagging, stop-word removal, tokenization, and others to become effective and natural [31-33]. Furthermore, Natural Language Generation (NLG) techniques such as content selection, document structuring, lexicalization, aggregation, linguistic realization, and others, provide chatbots the ability to actually generate natural human-like language [34].

\subsection{Knowledge Graphs}

Knowledge Graphs (KGs) concern a new AI technological trend that has its origin to Knowledge Bases (KBs) and the Semantic Web (SW). A KG (i) describes real world entities and their interrelations, organized in a graph structure, (ii) defines classes and relations of entities in a schema, (iii) allows the interrelation of entities to each other and (iv) covers various domains. A KG is an effective and efficient way of representing a KB of interconnected descriptions of real-word entities and events. KGs are very flexible data structures providing chatbots unlimited access to vast stores of knowledge $[15,29,35]$.

\subsection{Semantic Web Technologies}

Semantic Web (SW) technologies enable people to create structured data/information stores on the Web, build commonly agreed and shared vocabularies, and write formal and explicit rules for handling interlinked data. Linked (open) data are empowered by SW technologies such as RDF [36], SPARQL [37], and OWL [38]. Resource Description Framework (RDF) and Ontology Web Language (OWL) are the two main descriptive Semantic Web technologies that play a crucial role in the formal representation and structuring of data, information, and knowledge, while SPARQL query language is used to express queries across diverse and federated RDF data sources. All these technologies play a crucial role in the formal representation, structuring and querying of data, information, and knowledge that chatbots must process in order to be able to deliver accurate machine-processable and machine-understandable conversations/dialogues.

\subsection{Human-Like Conversational AI}

Chatbots or so called 'conversational agents' are interactive user interfaces that mimic human-to-human communication using NLP, ML, and other AI technology [39]. For chatbots to behave like humans they must be able to conduct human-like conversations. This can be achieved when human-like features and behaviors are included in the conversation with a human. Informal language, personalization, consciousness, intentions, irony, sentiments, and other behavioral and linguistic characteristics must be included in the design of dialogues. In addition, human-agents interacting with chatbots must be allowed to ask free-text questions in contrast to (always) following a predefined dialogue path using buttons and predefined questions. Chatbot dialogues that either lead to dead-end conversations or respond in inappropriate way because of inability to recognize, for instance, the tone of a question, or respond to a first-person question with a third person answer, is considered a non-human-like dialogue [40].

A human-like interaction is mainly generated in the case where a chatbot learns from previous discussions/interactions, utilizing past knowledge with fine conversation patterns and meaningful dialogues [41]. Anthropomorphism is the desirable trait that chatbots must have so user-friendly chatbots will be able to engage and build a social relationship with humans [42]. However, conversational human-like AI cannot be easily deployed; there are several technical factors and implications, in addition to ethical and/or legal issues such as biased training, users' misbehavior, content violations, etc., inevitably leading the research community to question the actual impact of fully human-like chatbots [41]. 


\subsection{Distributed Collaboration in Multi-Chatbot Systems}

In distributed collaboration, human, software, and computer agents work together to reach a certain goal $[43,44]$. The concept is not new and is implemented in many domains and systems where the complexity of the operation is requiring a system architecture that is flexible, integrates autonomous functions, and delivers fast and reliable responses to end-users. Internet infrastructure, emails, instant messaging, GIS systems, gaming, virtual meetings software, crowdsourcing platforms, etc., rely on the concept of distributed collaboration [45]. In conversational AI domain, chatbots need to collaborate towards achieving a common goal, i.e., discover the knowledge required for a question to be answered in a dialogue, and then deliver this knowledge in the right form. Distributed knowledge is not encoded in the single $\mathrm{KB}$ of a single chatbot per organization or application domain, but it is distributed in several smaller pieces (KGs) to facilitate a more efficient and flexible architecture where every chatbot in a network (group) of chatbots per organization is responsible to provide a small chunk of knowledge [46].

IBM research introduced COBOTS in 2018, a cognitive multi-bot conversational framework that uses IBM Watson chatbot platform. A multi-chatbot system architecture was presented, where an orchestration chatbot gets the users intent, identify it and routes it to the suitable expert chatbot that searches for the right answer in its KG. Although COBOTS architecture is distributed, there is no clear report that the expert chatbots can collaborate with each other or with other chatbots [47]. OpenDialog chatbot platform is focusing on developing conversations that are imitating real human conversations. In their manifesto, OpenDialog developers are stating that chatbot conversations must have a purpose, must be dynamic, non-linear, and multi-faceted and must take into account all participants goals, biases and motivations. They also state that a conversational system must be able to have multiple human participants and multiple chatbot agents that can collaborate under a host chatbot supervision. The latest edition of OpenDialog chatbot platform is moving towards adopting the distributed collaboration concept [48]. Rasa chatbot platform developers acknowledge the need of a chatbot that knows and answers everything, so they introduce the concept of meta-bots [49] and multi-bot orchestration [50], where multiple chatbots are developed to function separately and simultaneously collaborate under one orchestration chatbot, facing the challenges of intent routing, context switching and server handling. Kore.ai chatbot platform also introduces the use of a universal bot that can be deployed to connect and orchestrate several other kore.ai chatbots. This solution covers only the distribution feature and assist the users to easily get their answer from the right chatbot. The collaboration feature however is not utilized at all levels and is retained in the upper level of serving the intent of the user [51]. Finally, ServisBot Company had introduced their multi-chatbot platform where an orchestration chatbot takes the role of the host and routes users' intent to any chatbot that is connected [52].

Distributed collaboration of chatbots is a real need. Intent routing, turn taking, context switching, server handling and fulfilling users' satisfactions are the major problems of developing a multi-chatbot system that adopt the distributed collaboration approach $[53,54]$. In addition, the collaboration feature is implemented with the use of a single orchestration chatbot that orchestrates the conversation and there is not always a direct collaboration between the distributed chatbots. Chatbot platform developers are still focusing their main research in developing more intelligent chatbot systems by exploiting and advancing the ML and NLP techniques. Distributed collaboration of multiple chatbots is a problem to be solved in the following years.

\section{State of the Art in AI Museum Chatbots}

Museums have been working with chatbot technology for more than a decade. The first chatbot-related applications were conversational bots interacting with the audience as avatars through screens or through phone or text services [5]. Gradually, with the rise of advanced AI technologies and the wide use of social media networking, more advanced chatbots were developed and used in several domains. Chatbots in museums are providing 
a new way of increasing visitors engagement by functioning as museum digital guides or as simple question/answering (QA) information agents or, in a more sophisticated way, as visit planners [6,8]. A systematic review of selective related efforts in developing chatbots for museums is provided in the following paragraphs. The review is conducted to examine the ways that chatbot developers and curators are designing the museum chatbots and exploiting the recent AI technologies, presented in the previous section.

\subsection{Description of Selective Museum Chatbots}

The following list of selected museum chatbots gives an overview of the current state of the art. It includes typical chatbots implementing various technologies, acting as a basis for the evaluation provided in Section 4.4. The detailed presentation of the selected museum chatbots supports their categorization and the identification of specific characteristics/attributes that are crucial for their evaluation and for the design of the proposed multi-chatbot architecture.

- At the National Art Museum of the Republic of Belarus, a simple QA Facebook Messenger chatbot has been developed with use of the Chatfuel chatbot platform [55]. The chatbot can answer simple questions about the artefacts of the museum and it can be identified as a simple infobot, with low quality conversational skills [56]. The bot was accessible on 30 August 2021 from https:/ / m.me/1904070043163955, but is currently inactive.

- At Anne Frank House in Amsterdam, an off-site Facebook Messenger chatbot was developed and implemented with the use of msg.ai platform, now established as Netomi chatbot platform [57]. The chatbot content is well curated as the user is guided to follow a predetermined route with the use of buttons through the life of Anne Frank and the museum's exhibits. The chatbot is always tries to be in control of the conversation. It is not designed to answer free questions and gets confused when this happens. The designed scenario follows the scope of the museum, is helpful for the users and safe for the museum as it provides protection from users' misbehavior [6,9]. The chatbot is currently inactive.

- A chatbot with the same features as the Anne Frank House chatbot is also implemented for the Museo di arte moderna e contemporanea di Trento e Rovereto [58]. The Martmuseum bot uses Facebook Messenger to guide visitors through its content with the use of predefined routes and was active on 30 August 2021 from https: / / www.messenger.com/t/martrovereto.

- The Maxxi's Chatbot is designed for the National Museum of the 21st Century Arts in Rome. The chatbot is developed with Google's Dialogflow and integrated in a Facebook page [59]. The Facebook Messenger chatbot uses predefined content and guides the users through word or image selections to follow a certain path in a guided route. The chatbot is lacking conversational skills but provides engagement through multimedia content, rewards and carefully designed dialogs [6]. The chatbot was accessible with a Facebook account on 30 August 2021 from https://m.me/ museomaxxi.

- At the House Museum of Milan an advanced Facebook Messenger chatbot was developed, named "Di Casa in casa" adventure chatbot. The chatbot is developed with the Wit.ai platform and uses gamification techniques in order to engage sole users or groups of visitors to play a "treasure hunt" tour game by finding clues and learning new things. The chatbot also tries to engage visitors by creating realistic non-linear narrative dialogue tours, though the chatbot has no free conversational skills $[5,6,9]$. The chatbot was active on 30 August 2021 from https://www.messenger.com/t/ casemuseobot, and is accessible only in the Italian language.

- At Catal Hoyuk Neolithic site an advanced Facebook Messenger chatbot, named ChatCat was developed by a group of experts. The development team of the ChatCat chatbot, after an extensive design and testing process on the content and the possible dialogues have chosen Wit.ai chatbot platform for the implementation of the chatbot. 
The reason for their choice was that Wit.ai is focusing on developing chatbots for the popular channel of Facebook Messenger, is supported by an active users' community and is providing a user-friendly interface for the development of the chatbot. The chatbot had the skill not only to provide useful content but also to provoke the user about a certain issue. The chatbot surprises its users, takes control of the conversation, and awakes emotions and thoughts. It is designed to make rule-based conversations that have a meaning, e.g., the chatbot can start a conversation about the concept of death in the Neolithic period. The final scope of the chatbot is to alter the perception of the users about an issue and engage them [8]. The developers of the ChatCat chatbot are letting the users to use their own words but try to limit their verbal interaction by controlling the conversation. If a user tries to be more creative in his conversation, the chatbot cannot understand and continuously tries to take control of the conversation. The chatbot was accessed with a Facebook Messenger account on 30 August 2021 from https:/ / www.messenger.com/t/catalhoyukbot, but it is currently inactive.

- The chatbot at The Field Museum of Chicago is representing the largest dinosaur relic and is called Máximo the Titanosaur. The chatbot is developed and trained with Google's Dialogflow platform. One of the main reasons that the developers chose Dialogflow was that the chatbot can be delivered to end-users via many channels (e.g., Facebook, Viber, WeChat, etc.). The developers designed a friendly, smart and delightful conversational chatbot, that is able to answer every possible question in a human-like manner. The developers used the NLU and ML techniques that Dialogflow provides, but the most important work was the continuous user testing and redesign of the possible dialogues. The developers shared the following concept: "For an agent that's meant to be exploratory and conversational, if it does not sound human, then it's just not...fun." [60]. Maximo was online on 30 August 2021 fromhttps: //www.fieldmuseum.org/exhibitions/maximo-titanosaur?chat=open, is designed to have a funny character and even to understand your sentiments. The chatbot was launched at May 2019 and by mid-October had 7.000 conversations with $72 \%$ accurate response rate [61].

- The Vincent Van Gogh Museum chatbot was designed to assist museum personnel to provide (via a Facebook page) useful information about the museum and Van Gogh artwork. The chatbot was developed with the open-source version of Microsoft Bot Framework and is entirely hosted on the Museum's paid Microsoft Azure cloud Server (paid version), providing total control over training, management, and development to the museum [62]. The chatbot is designed with the aim to be conversational, as it allows free questions, however, it cannot successfully complete a human-like conversation. The chatbot identifies the user's intent and provides text information and web links. The chatbot can be classified as an infobot and was accessible on 30 August 2021 from https:/ / www.facebook.com/messages/t/127104175596.

- Andy Carnegie Bot is a digital character that guides visitors to summer Carnegie Museums of Pittsburgh. Andy also provides updates on events and museum activities. The chatbot was developed with Botpress open-source platform [9,63]. The chatbot is using a gamification process with QR codes and tries to gain visitors engagement through a predefined treasure hunt game that helps visitors collect stamps digitally [64]. The developers of Andy did not focus to engage the visitors through human-like conversations. Instead, they chose to brand the chatbot with interesting graphics, fun visual and text elements, and a fun hunting game. The development team conducted large-scale research to reach to the final decisions for the design and the development of Andy Carnegie Bot [65]. The chatbot was active on Facebook Messenger only on summers (30 August 2021) from https:/ / www.facebook.com/carnegiebot/.

- The Museum of Tomorrow at Rio de Janeiro is a newly established science museum with futuristic architecture. Its main exhibition presents to the public a structured narrative of 5 science areas: Cosmos, Earth, Anthropocene, Tomorrow and Us, where 62 science experiments are presented, curated with high technological applications. 
One of these applications is IRIS, a chatbot that is installed inside the museum in several screen spots. The visitors receive at their entrance a smart card and anytime they spot an IRIS screen they can talk to it. IRIS is developed with IBM Watson framework and is designed as a chatbot that uses NLU and ML techniques to provoke the users to conduct a dialogue about certain humanity issues and express their concerns. At the end of the dialog IRIS suggests a related local initiative that visitors can participate. The chatbot was trained for many months with the use of testing teams and several testing methods. IRIS speaks Portuguese and English and is mainly a verbal chatbot that interacts via spoken words. However, due to the need of serving hearing-impaired visitors, a text input interface version had been also developed. In addition, IRIS collects all the concerns of the visitors and visualize them in a big screen constellation of colored particles that connect randomly, with a subtle, in a continuous motion. For this functionality the chatbot uses the IBM Bluemix cloud platform, where all the knowledge can be collected and visualized appropriately [9,66-69]. IRIS chatbot is only accessible to the visitors of the museum and it cannot be evaluated. A video presentation was available on 30 August 2021 from https: / / www.youtube. com/watch?v=0944e3x-hq4

- The museum of Modern Art at Buenos Aires developed the "Dialogue with the artwork" chatbot. The chatbot is designed with NLU and ML techniques and its innovation is that the visitor could chat with each artwork when she/he was in front of it. For each artwork a different chatbot with its own artistic character was developed. The chatbots are accessible through Facebook Messenger and could conduct smart, funny, and informative human-like dialogues [70]. There is no further information about this chatbot and is currently not active. However, the idea of developing a chatbot for each artwork is rather intriguing and aligned to a distributed and collaborative chatbot architecture proposed in this paper.

- Pinacoteca of São Paulo developed in 2017 the "Voice of Art" chatbot [9,71]. The chatbot is a native mobile voice chatbot developed with IBM Watson and IBM Bluemix cloud platform. The chatbot is using NLU and ML techniques and its training lasted around one year. The users can chat with seven artworks of the museum. The artworks communicate with the mobile app via smartphone beacon sensors and Bluetooth geolocation technology. The knowledge was stored in IBM Bluemix cloud platform and was collected from various sources. The chatbot was able to answer verbally questions about the artwork and do it in human-like manner [72]. The idea of developing a chatbot for each artwork is aligned to a distributed and collaborative chatbot architecture proposed in this paper. "Voice of Art" chatbot is only accessible to the visitors of the museum and it cannot be evaluated. A video presentation was available on 30 August 2021 from: https:/ / www.youtube.com/watch?v=ogpv984_6 $0 A \& \mathrm{t}=185 \mathrm{~s}$.

- Ask Mona Studio is a startup chatbot company that had developed around 44 chatbots for museums and cultural organizations in France [73]. There are no clear descriptions in the bibliography of the technologies that Ask Mona Studio is using. It appears that a chatbot platform similar to Chatfuel has been used, a platform that develops simple predefined QA Facebook Messenger chatbots [74,75]. The designed chatbot conversations are predefined and curated with multimedia material. Users must follow a certain path, and every time they try to place a free-text question, the chatbot answers with a predefined answer or a message/reply of ignorance [76]. One of the chatbots, Ask Sarah chatbot at Petit Palais-City of Paris Fine Art Museum was available and active in Facebook Messenger on 30 August 2021 from https: / / www. messenger.com/t/273861966942/.

- The Culture Chatbot project [77] is an EU funded project that is initiated by the Jewish Heritage Network [78] and is implemented in three museums: The Museum of History of Polish Jews (POLIN) in Warsaw [79], the Jewish Historical Museum in Amsterdam and the Institute for the Union Catalogue of Italian Libraries and 
Bibliographic [80]. The project has researched the available (at that time) chatbot technologies and reached to three types of that could be implemented in the three partner museums: free-text search chatbot, guided search chatbot and engagement search chatbot. The platform that was chosen for developing the chatbots was Rasa chatbot platform [81]. The chatbots were developed to use NLU and ML techniques and designed to deliver a human-like conversation. In addition, they were designed to be connected to knowledge graphs [82]. The chatbots can be integrated in a webpage or a Facebook page and are bilingual. One of this chatbots was available in the Jewish Historical Museum in Amsterdam on 30 August 2021 from http:/ / chatbot.jck.nl/ and is a guided search chatbot. The chatbot is not fully trained yet and cannot answer freetext questions in a human-like manner. In addition, it is not clear in the bibliography that a KG was developed and implemented.

- The Cartier Foundation for Contemporary Art in France developed a chatbot that is available at https: / / www.fondationcartier.com/en/ (30 August 2021). The chatbot was developed with Google's Dialogflow and was designed to work with predefined questions and answers. The chatbot allows users to place their own free-text questions [83]. The chatbot can understand the intent of the questions but its training is limited. Irrelevant questions are answered with a predefined answer or an answer of ignorance and the chatbot tries to control/guide the conversation by providing to the users predefined multimedia content [84].

- The Akron Art Museum in Cleveland developed a fun Facebook chatbot named Dot $[9,85,86]$. The Dot is a guide chatbot with a well curated guided tour dialogue. Dot is friendly, fun, and smart but not clever enough, since it cannot answer any free question. However, Dot manages to keep up the interest and the excitement of museum's visitors with her "character" and the well-curated content and dialog design. Dot was accessible on 30 August 2021 from https:/ / www.messenger.com/t/ 380485969025647.

Based on the related efforts on museum chatbots listed above, we distinguish five types of chatbots in the museum domain. The criteria for this categorization are: (a) the conversational skills, and (b) the techniques that the chatbot developers use to engage the users. These types are:

(a) Simple QA informational chatbots (infobots), that can only provide simple information about the museum and its collections, with limited conversational skills. An example of an infobot is the National Art Museum of the Republic of Belarus QA Facebook Messenger chatbot.

(b) Chatbots with predefined routes of conversation, where the users get information about the museums by following predefined conversation routes with the assistance of text or image buttons. Their conversational skills are limited. The chatbots at Anne Frank House in Amsterdam and the National Museum of the 21st Century Arts in Rome are classified in this type of chatbots.

(c) Gamification and reward chatbots, where the users also follow predefined routes of conversation but the chatbot developers try to engage them with rewards and gamification features like a treasure hunt. Their conversational skills are limited. Maxxi's Chatbot and the chatbot at the House Museum of Milan are chatbots that use gamification techniques and rewards to engage users.

(d) Provoking conversational chatbots, where the chatbots try to control the conversation and provoke the users to ask questions that concern certain information. These chatbots have good conversational skills. Two examples of this type of chatbots are the Catal Hoyuk Neolithic site chatbot and the IRIS chatbot at Museum of Tomorrow at Rio de Janeiro.

(e) Fully human-like conversational chatbots, where the users can freely ask almost anything, without following any rules or predefined routes. The chatbots have the ability to understand the user's intent and provide most of the times the right answer. The Máximo the Titanosaur chatbot at Field Museum of Chicago is such a chatbot. 
In a previous line of research [87], only three types of chatbots were identified. We extend this categorization with provoking conversational chatbots type, as provocatory conversation is a new significant feature added in chatbot technology to differentiate from all other types. We also extend the categorization with a fifth type, the fully human-like conversational chatbots, as those are exploiting advanced ML and NLU techniques, as well as KGs, to elevate conversation at near-human levels.

A decisive factor that determines the type of chatbot that a museum chooses to develop is its budget/funding. Museums typically develop the type of chatbots they can afford. A fully developed AI conversational chatbot requires a lot of effort, support, training, and funding. The survey we have conducted also reveals that one of the main challenges of the museum stakeholders and the developers is to decide whether the designed chatbots will stay simple (e.g., with predefined QA) or will follow the recent trends, become smarter, use advance AI technologies, and engage audience through meaningful human-like (nearhuman intelligence) conversations $[9,25,88]$.

\subsection{Evaluation of Museum Chatbots}

The aim of our work is to first evaluate a set of representative museum chatbots in a way that useful insights and technical knowledge will be highlighted and then utilized for an efficient design of the distributed, collaborative, and knowledge-graph-based conversational AI multi-chatbot architecture, as proposed in this paper. Such an evaluation must be conducted with a methodology that follows certain qualitative attributes. Nicole Radziwill and Morgan Benton had conducted a related survey, extracting the qualitative attributes of chatbots in different domains [89]. They concluded that a chatbot evaluation methodology should contain qualitative attributes that align with the ISO 9241 concept of usability, defined as "The effectiveness, efficiency and satisfaction with which specified users achieve specified goals in particular environments" [90]. They finally presented in the "Quality Attributes" section of the article, a detailed table of the qualitative attributes organized in terms of ISO 9241 standard, [89].

There are a number of qualitative attributes selected for the evaluation of museum chatbots, derived from the extensive study of related works and from the hands-on experience on active chatbots. Selected attributes from the abovementioned table are used in the evaluation of the museum chatbots. Beyond the attributes used in other evaluation reports/papers, the following qualitative attributes, focusing on a distributed, collaborative and KG-based system architecture, are examined:

(a) Integration of KGs for enhancing the precision and coverage of conversations;

(b) Distributed knowledge facilitation via multi-chatbots and KGs;

(c) Multi-chatbots collaboration for knowledge acquisition and delivery.

\subsection{The AHP Evaluation Method}

The Analytic Hierarchy Process (AHP) evaluation method was established by Saaty [91] to provide a powerful and flexible decision-making process to prioritize a set of quality attributes. The AHP method is widely used in the field of information systems as its main advantage is that the users of the AHP method can determine their own evaluation criteria (attributes) for the evaluated systems. The AHP method comprises in short of six main steps [91-94]:

1. State the problem and identify all the attributes and sub attributes that determine the problem;

2. Structure the hierarchy of different attributes;

3. Compare each attribute pairwise in its hierarchy level and measure them on a qualitative and quantitative numerical scale (1/9: least valued to 9/9: most important);

4. Calculate the weights of each attribute and place them in a comparison attribute matrix;

5. Determine the final comparison weight of each attribute;

6. Conduct the evaluation. 
The Analytic Hierarchy Process (AHP) evaluation method to evaluate both the selected museum chatbots as well as the chatbot platforms is presented in the following section.

\subsection{Evaluation Process}

The evaluation of the selected museum chatbots followed comprises the following three steps:

1. Select chatbots that have conversational skills;

2. Select chatbots that integrate KGs;

3. Evaluate chatbots using the proposed quality evaluation attributes and the AHP evaluation method.

In Tables 1 and 2 we identify certain characteristics that each of the presented chatbot are fulfilling or not, in order to examine whether the chatbots can be evaluated with the use of the proposed quality evaluation attributes and the AHP evaluation method. In these tables we have not included the distributed collaboration feature, as all the museum chatbots that we have researched are designed following a single-chatbot architecture. For two of the museum chatbots, i.e., the Dialogue with the artwork chatbot (Buenos Aires) and the Voice of Art Chatbot (Pinacoteca of São Paulo), there is an indication of operating in a distributed manner, but there are no enough evidences to adequately support their claim. The first two (selection) steps result in a filtered/reduced evaluation list (Section 4.1).

In particular, the chatbots that are described as conversational chatbots (provoking conversational bots and fully human-like conversational chatbots), are outlined in the following list:

1. Máximo the Titanosaur Webpage chatbot;

2. IRIS at Museum of Tomorrow at Rio;

3. Dialogue with the artwork chatbot (Buenos Aires);

4. Voice of Art Chatbot (Pinacoteca of São Paulo);

5. EU Culture Chatbot project;

6. Cartier Foundation for Contemporary Art chatbot.

From these six conversational chatbots, three of them (number 2, 3 and 4) are not accessible through a webpage and cannot be further evaluated with a hands-on experience. Chatbots 1, 5 and 6 are accessible, however, we did not proceed to their evaluation with the use of the AHP evaluation method, as chatbots 1 and 6 do not integrate KGs, the main key feature of the proposed architecture. Finally, although chatbot 5 is designed to use KGs as a knowledge source, it is not clearly stated on the bibliography that there were used in its final implementation.

The conducted research and evaluation of chatbots reveals that, although the use of KGs and the distributed collaboration approach appears as challenging for developers and museum stakeholders, it is difficult, mostly for technical reasons, to be easily implemented in a museum chatbot solution. 
Table 1. Selected Museum chatbots' characteristics (I).

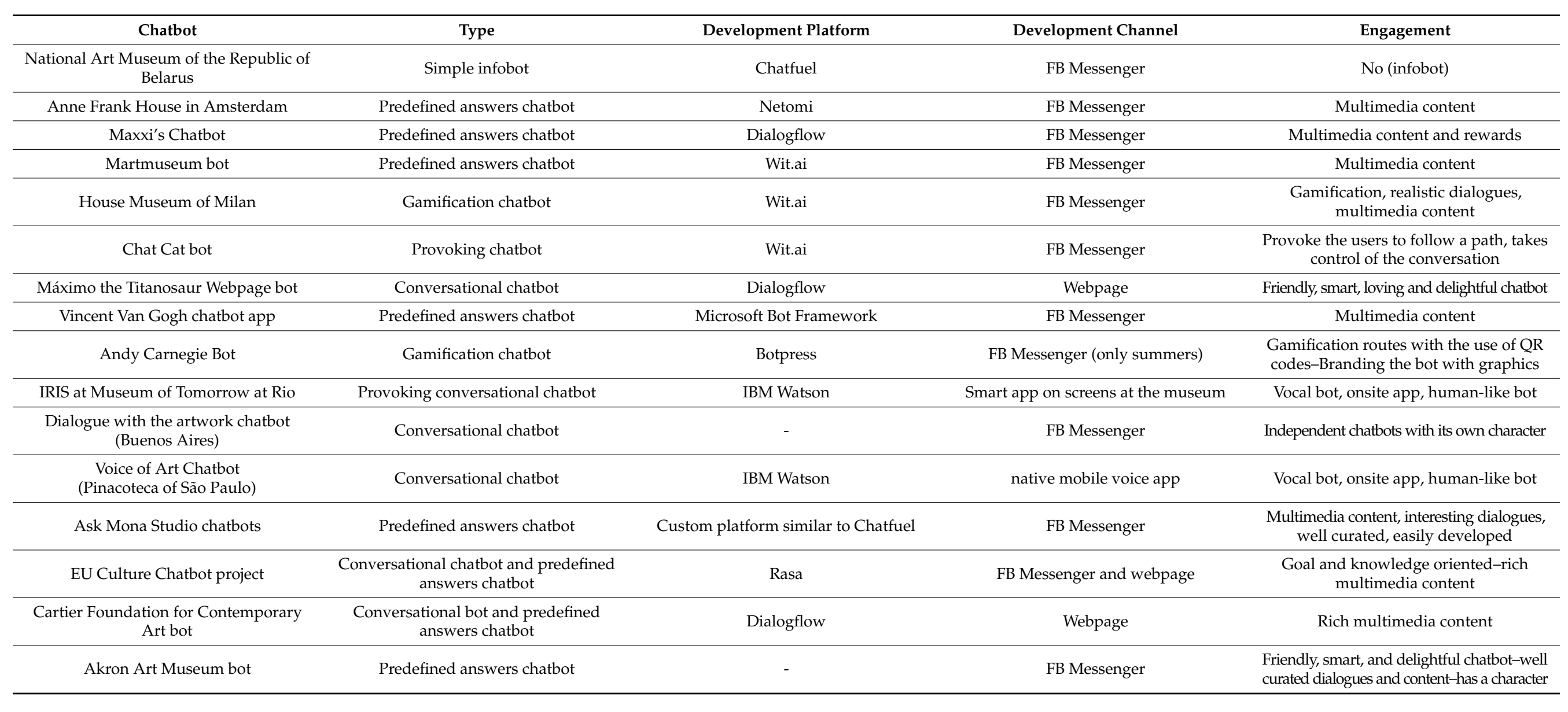


Table 2. Selected Museum chatbots' characteristics (II).

\begin{tabular}{|c|c|c|c|c|}
\hline Chatbot & Conversational Skills & Advanced AI Methods & KGs Support & Active \\
\hline $\begin{array}{l}\text { National Art Museum of the } \\
\text { Republic of Belarus }\end{array}$ & No & No & No & No \\
\hline $\begin{array}{l}\text { Anne Frank House in } \\
\text { Amsterdam }\end{array}$ & No & No & No & No \\
\hline Maxxi's Chatbot & No & No & No & Yes \\
\hline Martmuseum bot & No & No & No & Yes \\
\hline House Museum of Milan & No & No & No & Yes \\
\hline Chat Cat bot & Rule based conversations & Limited NLU training & No & No \\
\hline $\begin{array}{l}\text { Máximo the Titanosaur } \\
\text { Webpage bot }\end{array}$ & Yes & $\begin{array}{c}\text { Fully trained, NLU and } \\
\text { ML }\end{array}$ & No & Yes \\
\hline Vincent Van Gogh chatbot app & Yes, not fully developed & Limited NLU training & No & Yes \\
\hline Andy Carnegie Bot & Available but not used & Available but not used & No & Yes \\
\hline $\begin{array}{l}\text { IRIS at Museum of Tomorrow } \\
\text { at Rio }\end{array}$ & Yes & NLU and ML & $\begin{array}{l}\text { Yes, on IBM Bluemix cloud } \\
\text { platform-Graph visualization }\end{array}$ & $\begin{array}{l}\text { Yes } \\
\text { onsite }\end{array}$ \\
\hline $\begin{array}{l}\text { Dialogue with the artwork } \\
\text { chatbot (Buenos Aires) }\end{array}$ & Yes & NLU and ML & No & No \\
\hline $\begin{array}{c}\text { Voice of Art Chatbot } \\
\text { (Pinacoteca of São Paulo) }\end{array}$ & Yes & NLU and ML & $\begin{array}{l}\text { Yes, on IBM Bluemix cloud } \\
\text { platform }\end{array}$ & Not defined \\
\hline Ask Mona Studio chatbots & $\begin{array}{c}\text { No } \\
\text { (Only predefined answers) }\end{array}$ & No & No & Yes \\
\hline EU Culture Chatbot project & Available but not used & Available but not used & Yes-Dgraph-not fully developed & $\begin{array}{l}\text { Yes, a } \\
\text { demo }\end{array}$ \\
\hline $\begin{array}{l}\text { Cartier Foundation for } \\
\text { Contemporary Art bot }\end{array}$ & Yes, but limited & Available but not used & No & Yes \\
\hline Akron Art Museum bot & No & No & No & Yes \\
\hline
\end{tabular}

\section{State of the Art in Conversational AI Platforms}

In the following paragraphs a review of the most well-known and widely used conversational AI platforms is presented, along with an evaluation based on the AHP method. The aim of this evaluation is to identify a platform that utilizes KGs and provide features that promote the distributed collaboration of multiple chatbots in a museum.

Based on our previous research, four categories of AI chatbot platforms were identified: (a) no-coding task-oriented platforms, (b) conversation-oriented platforms, (c) conversational AI platforms backed by tech giants, and (d) conversational AI platforms backed by startup companies [95]. More specifically:

(a) The no-coding platforms are simple task-oriented platforms that do not integrate advanced AI (NLU/ML) capabilities. Examples of the famous platforms in this category are Chatfuel [96], Octane.ai [97] and Motion.ai [98].

(b) The conversation-oriented platforms are not designed to serve a specific task but to develop chatbots that follow a specific conversation with humans (or software agents), with no use of advanced AI techniques. An example of the most famous platforms in this category is PandoraBots [99].

(c) The conversational AI platforms backed by tech giants are the most advanced platforms that are using advanced AI techniques (NLU and ML) and integrate large knowledge sources such as webpages, ontologies, KGs and others. Examples of the most famous platforms in this category are Google's Dialogflow [100], Facebook's Wit.Ai [101], IBM's Watson Assistant [102], Microsoft's Bot Framework [103] and Amazon's Lex [104]. 
(d) The conversational AI platforms backed by startup companies are also platforms that use advance AI techniques and can integrate large sources of knowledge. The most famous platforms in this category are Rasa Framework [105], Botpress [106] and OpenDialog [107].

In the following paragraphs a description of the ten most famous (well-known and widely used) platforms of the last two categories, i.e., (c) and (d) is provided. The museum chatbots that are presented in this paper were developed with one of these platforms.

\subsection{Conversational AI Platforms Backed by Startup Companies}

\subsubsection{Botpress (BP)}

Botpress is an open-source framework written in JavaScript (Node.js.). Botpress provides a user interface where developers can design and manage their chatbots after their deployment. Developers can fully customize chatbots, use built-in modules such as QA, analytics, flow editor and NLU, add business logic, or integrate third party APIs. Chatbots developed with Botpress can be deployed on Facebook, Slack, Telegram, Twilio, and on websites. Botpress framework is a dual-license framework (free and professional edition). The Botpress framework does not support by default the integration of KGs but provides flexibility to integrate them via API calls [106,108]. Furthermore, there is no clear documentation whether the chatbots developed with Botpress could collaborate and continue to work in a distributed way. This architectural concept is not included in the initial concept of the platform.

\subsubsection{OpenDialog (OD)}

OpenDialog is an open-source framework for developing conversational applications to automate, scale and improve processes and interactions between humans and machines. OpenDialog is a Laravel application with a MySQL backend for user management and content management. OpenDialog uses NLU and ML techniques and can be connected to KG databases to store conversations and structure organizational knowledge. Specially, OpenDialog uses the Dgraph backend (a graph-based database written in Go) [109]. OpenDialog can be easily integrated in many different channels such as, spaCy, Facebook Messenger, WhatsApp and others. Additionally, it can communicate and collaborate with other chatbot platforms such as Google's Dialogflow, Amazon's Lex, Microsoft's Bot Framework, Rasa and others. Open dialog has recently released its updated beta version 1.0. with the aim to enrich its documentation and expand its user's community support. [107]. OpenDialog developers are stating in their manifesto that their platform is providing to the users the possibility to design multi-chatbots systems, which are considered autonomous entities that can collaborate with other chatbots and humans to solve common problems [48].

\subsubsection{Rasa Framework (RF)}

Rasa is an open-source conversational framework written in Python. It has two main open-source components: Rasa NLU and Rasa Core. Rasa Enterprise is a paid and more advanced version of Rasa platform. Rasa core allows more sophisticated dialogues trained by using interactive and supervised ML. Rasa core supports Facebook Messenger, RocketChat, Slack, Telegram, and Twilio. Rasa NLU component is responsible for the natural language understanding. Rasa can be connected with knowledge graph databases such as Grakn [110], Neo4j [111], OrientDB [112], GraphDB [113] and provide more specific and accurate knowledge to end-users. Additionally, developers can add different spoken languages to the framework. Rasa framework provides a strong documentation and a vivid open-source community. Rasa does not (currently) provide a user interface to its opensource version. A user interface is available with the Rasa X component installation and with the enterprise version [105,114-118]. Furthermore, Rasa can support the distributed collaboration architecture and is allowing users to develop systems with multiple bots, which are named as metabots or multi-chatbots. However, the developers are facing many challenges, such as intent routing, context switching and server handling [49,50]. Recently, 
Botfront.io platform (available from 30 August 2021, https:/ /botfront.io/) was developed by Rasa community as a front-end that provides a sophisticated UI for the development of Rasa chatbots [119].

\subsubsection{SnatchBot (SB)}

The SnatchBot platform is designed to support the entire lifecycle of a chatbot, from developing and testing to deploying, publishing, hosting, tracking, and monitoring. The platform provides robust administrative and technical features that include NLU, ML and voice recognition. Its advantage is that advanced coding experience is not required for developers, who can even deploy a chatbot through ready-made chatbot templates. Chatbots can be deployed for free via SMS, Website, FB Messenger, Twitter, Slack, WeChat, Skype, and other channels. The chatbots can be connected through JSON APIs with external knowledge databases or KGs. SnatchBot architecture does not support the concept of a distributed and collaborative system of chatbots, as their basic concept design is to provide a robust chatbot solution that guides the users to the right choice and answer [120]. SnatchBot has a free version and a paid one with a variety of plans. SnatchBot is a quality solution for the easy/simple development of chatbots. The developers can write their own NLP models and train them via platform's functionality and interface. There is strong documentation with articles, video tutorials and community support. The platform provides limited access to its source code [121,122].

\subsubsection{Kore.ai (Kai)}

Kore.ai is a business oriented dual license conversational AI platform that supports the development of chatbots without coding expertise. Kore.ai adapts to conversations in realtime, using NLU techniques. Kore.ai can be deployed through many channels such as SMS, Website, FB Messenger, Twitter, Slack, WeChat, Skype, and other channels. Kore.ai also supports the development of AI functions that can be used for extracting knowledge from documents, databases, and others. It also has its own KG infrastructure that helps users convert static FAQ text into a KG and lead to an intelligent, personalized conversational experience. The user can develop a hierarchical structure of key domain terms and associate them with context-specific questions and their alternatives, synonyms, and ML-enabled classes. This structure, when trained by the platform, generates a Knowledge Graph that enables an intelligent FAQ experience [123-125]. In addition, the Kore.ai developers are supporting a distributed and collaborative system of chatbots that are coordinated by a new universal chatbot that routes the user's intents to the proper independent chatbot [51].

\subsection{Conversational AI Platforms Backed by Tech Giants}

\subsubsection{Dialogflow (DF)}

Dialogflow (standard edition) is a conversational AI platform backed by Google that provides human-computer interaction in a voice or text-based conversational interface and strong NLU in 20+ languages, utilizing techniques such as intent classification, entities extraction, keyword extraction and sentiment analysis. Dialogflow provides a REST API which can be used to integrate chatbots to any application or to custom conversational interfaces. It supports all the major messaging channels such as Facebook Messenger, Slack, Skype, Kik, Line, Telegram, Twitter, Viber, etc. Dialogflow can be easily connected with a Knowledge Graph back-end such as Grank.ai. Dialogflow can communicate with Grank.ai or any Knowledge Graph backend, by using a webhook to a Rest endpoint. Furthermore, Dialogflow can deploy chatbot systems that support the distributed and collaborative architecture, as it allows the development of a "Mega" Agent, where developers can combine multiple Dialogflow agents-chatbots. When a user set a question the "Mega" Agent is designed to recognize the intent, search all the connected chatbots and provide the right answer [126]. Dialogflow has two editions: a) the standard edition which provides unlimited text messages for chatbot applications and Google assistant, with limitations in 
speech recognition and the amount of knowledge connectors, $b$ ) the Pro edition that offers text and audio messages with a cost per request $[92,100,117,118,127,128]$.

\subsubsection{IBM Watson Assistant (IWA)}

IBM Watson Assistant is a cloud service that allows software developers to embed an AI virtual assistant (VA) in any application, device, or channel. Watson Assistant uses Watson AI ML and NLU in 13 languages. It is mostly marketed to businesses that want to have the option of keeping private their data that flows through their virtual assistant. In addition, Watson Assistant can develop an artificial personality that reflects the business needs. The use of Knowledge Graphs and databases is integrated with the use of APIs. Additionally, developers can combine their independent chatbots by creating an agent or interface chatbot that orchestrates the flow of the user's intents. This distributed and collaborative architecture is not supported officially by IBM, but developers are able to implement it [47,129]. IBM offers free (limited), standard, and premium plans for the Watson Assistant. Watson Assistant is designed to work perfectly with the internal components that IBM is offering, such as IBM cloud [92,117,118,123,127,130,131].

\subsubsection{Facebook Wit.ai (Wit)}

Wit.ai is a free open-source conversational AI platform backed by Facebook. It has built-in NLU features to understand intents, entities extraction, actions, context, and offers support for over 137 languages. The developers can develop and deploy chatbots in many different channels. Wit.ai provides SDK in Node.js, Python, Ruby, and HTTP API. The use of Knowledge Graphs and databases is integrated with the use of APIs [101,118,127,132-134]. There is no reference that wit.ai is supporting a distributed and collaborative multiple chatbots architecture, though it cannot be excluded by developers with the use of thirdparty components.

\subsubsection{Microsoft Bot Framework (MBF)}

The Microsoft Bot Framework consists of Bot Framework SDKv4 and was developed to enable developers to model conversations and build chatbots using. NET, C\# and Node.js. With the help of the Azure Bot Service, it can be deployed on many channels, such as Skype, Cortana, Facebook, Messenger, a standalone website, etc. The framework has support for speech and intents understanding by connecting with Microsoft Language Understanding Intelligent Service (LUIS). It can use KG databases with API connectors. Microsoft Bot Framework does not officially support the proposed distributed and collaborative architecture, but developers had implemented solutions that combine separate chatbots with the use of an orchestration bot $[135,136]$. Microsoft Bot Framework provides a standard free version with unlimited messages on standard channels (Cortana, Skype, and Microsoft Teams) and limitation on premium channels, which can be unlocked with a subscription per number of messages. The Microsoft Bot Framework is considered a complex all-purpose platform that requires much effort in combining many different sub-apps $[92,103,117,123,127]$.

\subsubsection{Amazon Lex (Lex)}

Amazon Lex is a Web service for building conversational interfaces into any application using voice and text. Amazon Lex provides the advanced deep learning functionalities of automatic speech recognition (ASR) and NLU, enabling lifelike conversational interactions. KGs can be integrated in the chatbot service either with the use of the internal component of Amazon Neptune, or with the use of an external API and Amazon Connect service [137]. The implementation of a distributed and collaborative architecture is not officially supported or stated by Amazon Lex, though it could be implemented with tools provided by Amazon Web Services or third-party modules. A unique in-house feature is that Amazon Lex provides the ability for multi-turn conversation. Amazon Lex chatbots can be published to messaging platforms such as Facebook Messenger, Slack, Kik, and 
Twilio SMS. Amazon Lex is free for a trial period with limited speech and text requests. After that free period or on the exhaustion of the free requests there is a payment per number of requests $[104,117,138,139]$.

\subsection{Discussion on the Presented Chatbot Platforms}

The conversational AI platforms described in this section provide sophisticated tools and modules to develop intelligent conversational chatbots.

The platforms developed by tech giants have strong technological support and provide features for the design of a fully functional conversational chatbot. However, some of them (Amazon Lex, Microsoft Bot, and IBM Watson) are complex systems that work perfectly with their built-in components and require an experienced IT team to fine tune them. These platforms can be considered as business-oriented platforms and high-cost solutions. Dialogflow and Wit.ai are user-friendly platforms and chatbots can be easily developed and deployed in several channels. Their main limitation is that they are strongly focused to the solution of their organization and community developers are not so keen in exploiting them in other ways.

On the other hand, the platforms developed by small startup companies have the advantage of providing a free, open-source edition, a simple user interface and developers can deploy them in any possible ways. Such platforms are OpenDialog, Botpress, and Rasa. Botpress and Rasa are supported by a strong user community that provide support and solutions for different types of user needs. OpenDialog is still in its first stages of development but has the same growth goals. These 3 platforms strongly depend on their user communities and require from developer's strong IT skills to develop and fine tune the deployed chatbots. SnatchBot and Kore.ai are non-open-source platforms, however, provide free access to most of their features through a sophisticated, fully functional user interface. Developers can easily design and deploy their chatbots and are supported by a strong community.

In the following paragraphs we conduct a detailed evaluation of these platforms, considering specific attributes driven by our extensive study of related works and the requirements that a KG-based, distributed, and collaborative multi-chatbot conversational AI system is posing.

\subsection{Platforms' Evaluation Using Analytic Hierarchical Process (AHP)}

The goal of this evaluation is the identification of the most suitable conversational AI platform for the design and implementation of a KG-based, distributed, and collaborative AI multi-chatbot conversational system in a museum. The platform must provide strong conversational skills and easily updated and rich knowledge sources. As it was stated in previous sections, this evaluation is conducted with the Analytic Hierarchical Process (AHP) evaluation method. Our evaluation approach is based on the methodology of Kostelnik [92] and Braun et al. [118] but is focusing on the museum domain and introduces different evaluation attributes.

\subsubsection{Evaluation Attributes}

The selection of the evaluation attributes was driven (a) by our extensive study of related works $[92,117,118,123,127]$ and (b) the design requirements of a KG-based, distributed, and collaborative multi-chatbot conversational AI system architecture. Such attributes comply with the needs of a medium-size museum, the main features of the examined platforms and the ability to be measurable and specifiable. Moreover, they comply with our research focus to design conversational agents that are based on KGs, they can collaborate to satisfy other agents (human or software) conversational needs and do this by utilizing available knowledge shared in a distributed manner. The specific attributes are:

1. Knowledge Graphs (KGs). Use of KGs for representing and integration of specific domain (museum) and general knowledge. 
2. Natural Language Understanding (NLU). Use of NLU techniques to interpret natural language (that humans understand) to formal language (that machines understand) and the opposite.

3. API interaction (API). It is essential for developing a system that does not rely only on trained conversations that are built in the platform, but can connect, search and extract knowledge from external sources and databases.

4. Distributed collaboration (DC). All the components of the system must be designed to function autonomously but at the same time to be able to collaborate using a multi-chatbot network. This attribute also allows the smooth transfer of the designed components of a chatbot to any other platform.

5. License (LS). Examine if the use of a fully open-source platform is an adequate solution, or a paid version is more flexible for the developers.

6. Multilingual support (MS). The platforms must provide multi-lingual support for the encoded conversations, especially in environments where users come from multilingual communities, as tourists visiting a museum. Moreover, the platforms must allow the extension to new languages.

7. Multiple channels support (MCS). The chatbots that are developed must be easily deployed via as many channels as possible, such as FB Messenger, WeChat, webpages, Twitter, Slack, Alexa, and others.

8. Machine Learning (ML). Use of ML techniques to train chatbots to understand simple or complex questions and provide the right answer with a high precision.

9. Additional capabilities (AC). In this attribute we can add the sentiment analysis, the ability of adding personality traits to the chatbot and other special features.

10. Text-to-Speech (TTS) and Speech-to-Text (STT). The platform must be able to translate speech to text and the opposite. The ability must be either an in-house ability or to be provided (via APIs) by external applications.

11. Self-configuration (SC). This attribute is controversial as self-configuration of chatbots is essential for museums with limited IT support to easily manage the deployed chatbots. On the other hand, this feature does not give the developers much flexibility to use or test other components and integrate them to the chatbot solution.

\subsubsection{Evaluation Process}

As abovementioned, for the evaluation of the platforms, we used the AHP evaluation method that was presented in Section 4.3. The first step is to calculate the weights of the attributes that were selected. This calculation is performed by comparing all attributes pairwise and the use of the hierarchy of comparison that the Saaty method is suggesting as is presented in Table 3 [91].

Table 3. Weights for attributes comparison using Saaty method.

\begin{tabular}{cc}
\hline Weight & Description \\
\hline 1 & Both attributes are equal \\
3 & Weakly preferred \\
5 & Strongly preferred \\
7 & Very strongly preferred \\
9 & Absolutely preferred \\
\hline
\end{tabular}

At this point the evaluation needs mathematical calculations to compare the attributes and calculate the final weights. For this reason, we used the AHP Priority Calculator that is developed by Goepel [140] and is accessible on 30 August 2021 from https:/ / bpmsg. com/ahp/ahp-calc.php [141]. From the use of the tool, we extracted Table 4 of pairwise comparisons. Each attribute is not compared with itself and takes the score 1 . The final column is demonstrating the weighted score for each attribute. These weighted scores will be used for the evaluation of each chatbot platform. 
Table 4. Calculating the weights of attributes for a museum.

\begin{tabular}{ccccccccccccc}
\hline & KG & NLP & API & DC & LS & MLS & MCS & ML & AC & TTS/STT & SC & Weight \\
\hline KG & $\mathbf{1}$ & 1.00 & 1.00 & 1.00 & 3.00 & 3.00 & 5.00 & 5.00 & 7.00 & 7.00 & 9.00 & $\mathbf{0 . 1 6 9}$ \\
\hline NLP & 1.00 & $\mathbf{1}$ & 1.00 & 1.00 & 3.00 & 3.00 & 5.00 & 5.00 & 7.00 & 7.00 & 9.00 & $\mathbf{0 . 1 6 9}$ \\
\hline API & 1.00 & 1.00 & $\mathbf{1}$ & 1.00 & 3.00 & 3.00 & 5.00 & 5.00 & 7.00 & 7.00 & 9.00 & $\mathbf{0 . 1 6 9}$ \\
\hline DC & 1.00 & 1.00 & 1.00 & $\mathbf{1}$ & 3.00 & 3.00 & 5.00 & 5.00 & 7.00 & 7.00 & 9.00 & $\mathbf{0 . 1 6 9}$ \\
\hline LS & 0.33 & 0.33 & 0.33 & 0.33 & $\mathbf{1}$ & 1.00 & 3.00 & 5.00 & 7.00 & 7.00 & 9.00 & $\mathbf{0 . 0 9 1}$ \\
\hline MLS & 0.33 & 0.33 & 0.33 & 0.33 & 1.00 & $\mathbf{1}$ & 3.00 & 5.00 & 7.00 & 7.00 & 9.00 & $\mathbf{0 . 0 9 1}$ \\
\hline MCS & 0.20 & 0.20 & 0.20 & 0.20 & 0.33 & 0.33 & $\mathbf{1}$ & 5.00 & 7.00 & 7.00 & 9.00 & $\mathbf{0 . 0 6 3}$ \\
\hline ML & 0.20 & 0.20 & 0.20 & 0.20 & 0.20 & 0.20 & 0.20 & $\mathbf{1}$ & 3.00 & 5.00 & 9.00 & $\mathbf{0 . 0 3 7}$ \\
\hline AC & 0.14 & 0.14 & 0.14 & 0.14 & 0.14 & 0.20 & 0.33 & 0.33 & $\mathbf{1}$ & 1.00 & 1.00 & $\mathbf{0 . 0 1 5}$ \\
\hline TTS/SST & 0.14 & 0.14 & 0.14 & 0.14 & 0.14 & 0.14 & 0.14 & 0.20 & 1.00 & $\mathbf{1}$ & 1.00 & $\mathbf{0 . 0 1 5}$ \\
\hline SC & 0.11 & 0.11 & 0.11 & 0.11 & 0.11 & 0.11 & 0.11 & 0.11 & 1.00 & 1.00 & $\mathbf{1}$ & $\mathbf{0 . 0 1 2}$ \\
\hline & & & & & & & & & & & $\mathbf{1 . 0 0 0}$ \\
\hline
\end{tabular}

The second step of the evaluation process is to score the attributes (criteria) of the platforms using the following scale on Table 5 [92]:

Table 5. Scores for evaluating individual attributes of platforms.

\begin{tabular}{|c|c|}
\hline Score & Description \\
\hline 1 & Support for this attribute is unavailable or this attribute does not apply \\
\hline 2 & $\begin{array}{l}\text { Some or very limited support for this attribute is available thanks to, e.g., } \\
\text { workarounds or other third-party software. }\end{array}$ \\
\hline 3 & $\begin{array}{l}\text { The attribute is available, but polishing is required. Has certain limitations that } \\
\text { affect its functionality or usability. }\end{array}$ \\
\hline 4 & $\begin{array}{l}\text { The attribute is available in the system, lacks some minor details such as complete } \\
\text { documentation or advanced functionality. }\end{array}$ \\
\hline 5 & Support for this attribute in the system is fully present and has no limitations. \\
\hline
\end{tabular}

In Table 6 we present the conducted evaluation by adding the scores to each attribute of each platform. Each attribute score was multiplied with its weighed score and all scores were added to produce the final score of the platform that is presented to the final line of the table.

Table 6. Evaluation of the platforms.

\begin{tabular}{ccccccccccccc}
\hline & Weight & \multicolumn{3}{c}{ Conversational AI Platforms Backed By Startup Companies } & \multicolumn{3}{c}{ Conversational AI Platforms Backed by Tech Giants } \\
\hline & & $\mathbf{B P}$ & $\mathbf{O D}$ & $\mathbf{R F}$ & $\mathbf{S B}$ & Kai & Lex & Wit & MBF & DF & IWA \\
\hline KG & $\mathbf{0 . 1 6 9}$ & 2.00 & 5.00 & 3.00 & 2.00 & 3.00 & 5.00 & 2.00 & 2.00 & 2.00 & 5.00 \\
\hline NLP & $\mathbf{0 . 1 6 9}$ & 5.00 & 3.00 & 5.00 & 5.00 & 5.00 & 5.00 & 5.00 & 5.00 & 5.00 & 5.00 \\
\hline DC & $\mathbf{0 . 1 6 9}$ & 4.00 & 5.00 & 5.00 & 1.00 & 1.00 & 3.00 & 2.00 & 2.00 & 3.00 & 1.00 \\
\hline ML & $\mathbf{0 . 0 3 7}$ & 5.00 & 2.00 & 5.00 & 5.00 & 5.00 & 5.00 & 5.00 & 5.00 & 5.00 & 5.00 \\
\hline TTS & $\mathbf{0 . 0 1 5}$ & 2.00 & 2.00 & 2.00 & 5.00 & 4.00 & 5.00 & 5.00 & 5.00 & 5.00 & 5.00 \\
\hline LS & $\mathbf{0 . 0 9 1}$ & 4.00 & 5.00 & 5.00 & 3.00 & 3.00 & 2.00 & 5.00 & 1.00 & 1.00 & 1.00 \\
\hline MS & $\mathbf{0 . 0 9 1}$ & 2.00 & 3.00 & 4.00 & 5.00 & 3.00 & 3.00 & 5.00 & 5.00 & 2.00 & 2.00 \\
\hline MCS & $\mathbf{0 . 0 6 3}$ & 5.00 & 5.00 & 5.00 & 5.00 & 5.00 & 5.00 & 1.00 & 4.00 & 5.00 & 2.00 \\
\hline API & $\mathbf{0 . 1 6 9}$ & 5.00 & 5.00 & 5.00 & 4.00 & 5.00 & 5.00 & 5.00 & 5.00 & 5.00 & 5.00 \\
\hline AC & $\mathbf{0 . 0 1 5}$ & 2.00 & 2.00 & 2.00 & 3.00 & 5.00 & 3.00 & 4.00 & 5.00 & 3.00 & 5.00 \\
\hline SC & $\mathbf{0 . 0 1 2}$ & 3.00 & 3.00 & 3.00 & 5.00 & 5.00 & 1.00 & 5.00 & 1.00 & 3.00 & 2.00 \\
\hline Weight Total & & 3.846 & 4.255 & $\mathbf{4 . 4 5 7}$ & 3.436 & 3.607 & 4.129 & 3.719 & 3.511 & 3.464 & 3.462 \\
\hline
\end{tabular}




\subsubsection{Results and Discussion}

The pairwise comparison (Table 4) of the attributes has been conducted by taking into account that most of the small or medium-sized museums cannot use or/and maintain a complex platform and also cannot fund a high-cost solution that tech giants mostly provide.

In addition, we considered that a platform must be able to provide strong conversational skills, utilize/integrate rich and reliable distributed knowledge sources, easily use API connectors, and have all its components independent, collaborative, and portable. For these reasons the attributes of KGs and NLU support, API integration and Distributed Collaboration were promoted (higher weight). Self-configuration would be considered as a useful attribute for some underfunded and understaffed museums, however most of the times this solution ends-up to abandonment. Support from an outside IT company could be preferable and eventually with lower costs.

The final weighted total score of the presented evaluation has determined that the preferable platform from the platforms backed by startup companies is the Rasa platform. OpenDialog platform is the second most preferable solution. SnatchBot and Kore.ai platforms though are providing easiness with self-configuration abilities; they lack flexibility and distributed collaboration.

From the conversational AI platforms backed by tech giants the highly scored one is Amazon Lex. Microsoft Bot, Dialogflow, and IBM Watson are very close in the score. However, all these platforms need strong technical skills and considerable costs after the free trial. Wit.ai is a free and preferable solution but is focusing to Facebook Messenger channel.

The conducted evaluation is focused on the needs of a small or middle range museum. A large organization such as the Acropolis Museum in Athens, that is highly funded and operates a strong IT department, can deploy a complex solution that is backed by a tech giant. Having said that, the AHP evaluation method is a flexible method that can be adjusted to any preferable needs in every domain and according to the goals of the development or research team.

\subsection{AI chatbots, KGs and advanced AI technology}

In this section, we present a selective list of chatbots that were developed with the presented platforms. The chatbots presented in Section 5.5.1. are using KGs for the representation of knowledge while the ones presented in Section 5.5.2. are exploiting advanced AI technologies such as ML and Deep Learning techniques. This review contributes to the identification of possible limitations, extensions, or different approaches in the development of chatbots using KGs and advanced AI techniques.

\subsubsection{KG-based AI Chatbots}

There are many proposed approaches to the recent bibliography that introduce KGs to AI chatbots and QA systems. Hallili [130] has proposed the SynchroBot, a dialog system that a) has connectivity to robust and flexible KBs and KGs for extracting in-formation, and b) could use NLP tools to interpret user's questions and NLG techniques to provide proper answers. This ability differs from other QA systems which are either focusing in providing a logical conversation ability to the users, without caring for the richness of their knowledge source, or, on the other hand, can only provide accurate answers without any conversation skills.

OntBot was an ontology-based chatbot that relied on the approach proposed by Hallili [142]. OntBot used Protégé [143] to develop an ontology template which gathers knowledge from e-commerce website APIs. Users can place their questions to a dialog manager that uses NLP such as Facebook Wit.Ai. The dialog manager connects to the ontology template with Python language, searches the KBs and KGs and provides the proper answer to the user with the help of NLU techniques from the dialog manager [144]. 
Disbot is a Portuguese Disaster Support Dynamic Knowledge chatbot that is implemented with Rasa Framework and is able to answer simple questions by exploiting NLP and ML training and also answer more complex questions that require knowledge extraction from a connected knowledge graph infrastructure. This KG infrastructure is designed to be dynamically updated with data from various sources such as social media and webpages [114].

NUBOT is also a KG-based chatbot that is developed with Rasa Framework and explores the implementation of a chatbot that speaks a dialect of the Pakistani Official Language, the Roman Urdu language. The researchers are focusing on the benefits of using KGs as their main knowledge source, on the difficulties in the NLU of the user's intent in Roman Urdu and on how to develop a system that performs better with less computational cost [116].

Another approach [134] is a chatbot developed with Wit.ai platform that is focusing on how to develop a KG-based chatbot that can assist users of an e-commerce website. The proposed architecture of the chatbot is focusing on how to convert data from e-commerce website APIs to XML/JSON format, store them in a Knowledge Base and then load them to a Protégé ontology template that can be accessed by the dialogue manager through NLP techniques and is able to retrieve the asked knowledge.

PolarisX-bot is a Dialogflow implemented chatbot that relies on KGs. The designers strongly suggest that KGs chatbots systems is the solution for human-like chatbots. Furthermore, they expand their use by introducing the auto-growing KG with the use of Googles BERT model. BERT model is designed to continuously extract knowledge from various sources that feeds and expands the KG of the chatbot. Their main challenge on their future work is the fine tuning of the relationships' extraction [128]. IBM researchers have designed and implemented an ontology-based chatbot with the IBM Watson Assistant. The researchers chose to develop an OWL ontology-based chatbot because their need was to develop a domain specific chatbot that will be able to provide robust and persistent context to its users. Their methodology relies on knowledge extraction techniques and in the assistance of domain experts which refine and train the chatbot system [130].

Finally, KBot is a KG-based chatbot that focuses on the integration of many Linked Data sources. The developers designed the system with Flask python framework and implemented with Chatterbot service. With the integration of NLU techniques, the system sets SPARQL queries to known Knowledge Bases and external web services. The extracted knowledge is gathered in a knowledge graph and with the help of a chatbot service the users receive their answer [145].

All the KG-based systems described above are focusing on the need to provide specific robust knowledge to the users, to develop chatbots that can understand the human language and to be easily updated with new (unlimited) knowledge. KG-based chatbots is the proposed and more effective solution, though there are many challenges mainly on combining NLU and knowledge extraction techniques.

\subsubsection{AI Chatbots Based on Advanced AI Technologies}

Samuel et al. 2020, have proposed a voice chatbot for bank payments with the use of Amazon Lex service. They exploited NLU, Automatic Speech Recognition and Speech Synthesis components that Amazon Lex service is providing and with the use of Raspberry Pi terminal, a microphone and a set of speakers implemented a task-oriented voice chatbot. Their main scope is to fine-tune the voice chatbot so it can assist mostly the visually impaired citizens [139]. Another approach is proposing the improvement of chatbot semantics with the use of Wit.ai platform and Word Sequence Kernel techniques. The approach is exploiting Wit.ai NLU techniques by comparing user queries to stored user queries and providing a more accurate response [133]. HOLMes chatbot system is an IBM Watson chatbot on the domain of healthcare recommendation. The aim is to design a health recommendation system which relies on ML, deep learning, and big data infrastructure, that can simulate a doctor-patient conversation that is reliable, inspires trust to the patients 
and is not biased [131]. Another approach [121] concerns a chatbot system for college inquiries that is implement with the SnatchBot platform and focuses on using NLP and ML techniques to set queries to a connected database server. The technique that is mostly used is keyword matching. Last (but not least) approach concerns a Botpress chatbot [108] that exploits Botpress platform in premises components to deliver a more intelligent chatbot that can answer complicated questions in a business environment.

\subsection{Discussion}

In Sections 3-5 we have presented a review of selected museum chatbots and conversational AI platforms. We have also discussed utilized technologies such as KGs, NLP, $\mathrm{ML}$, and also a distributed collaboration approach and its role in the development of multichatbot systems. Finally, we have presented a review on chatbots that were developed with selected platforms and a number of AI techniques.

The evaluation of museum chatbots and conversational AI platforms has been focused on examining whether KG support and distributed multi-chatbot collaboration can be easily implemented and how. This research concludes that although KGs and distributed multi-chatbot collaboration is not fully supported and implemented in current research and development projects, the community is now investigating ways towards this realization, developing advanced tools and methods for this to be possible in the near future.

\section{The Proposed Architecture}

Based on the survey conducted in this line of research and on our previous work [16], this section presents the proposed architectural design of a KG-based, distributed, and collaborative multi-chatbot conversational AI system for museums (Figure 1 depicts an example instantiation of the proposed system architecture). The proposed architecture is promoting the concept of using a network of distributed and collaborative chatbots (multi-chatbot system) within an organization. Each chatbot in the network has its own aim and scope, a specific thematic/domain conversation flow, its own knowledge, and it can also collaborate with other chatbots in the network, share and retrieve knowledge in a distributed manner. Chatbots in such an architecture can provide autonomous conversational services (based on their skill), and at the same time they are able to collaborate to support complex knowledge acquisition and delivery tasks for the museum visitors. Additionally, our approach will adapt the orchestration-by-host-chatbot solution as it was presented in Section 3.6, taking into account that this structural choice is currently considered by platform developers the most suitable technological approach for distributed multi chatbot collaborative solutions.

The proposed architecture (Figure 2) relies on five main components:

(a) A chatbot service that provides access to the distributed and collaborative chatbots of any kind and number. The chatbot service must be available through many channels (e.g., Facebook, Viber) and devices (desktop, tablet, smartphone).

(b) A knowledge index store mechanism that identifies the user's intent and selects the appropriate chatbot that can provide specific knowledge to specific visitors.

(c) An NLP component for transforming user input from natural to formal language

(d) A KG component that utilizes Semantic Web technology (RDF, SPARQL query language, and OWL ontologies) for the representation, linking, reasoning, and querying of knowledge.

(e) An NLG component for generating well-defined human-like answers. 


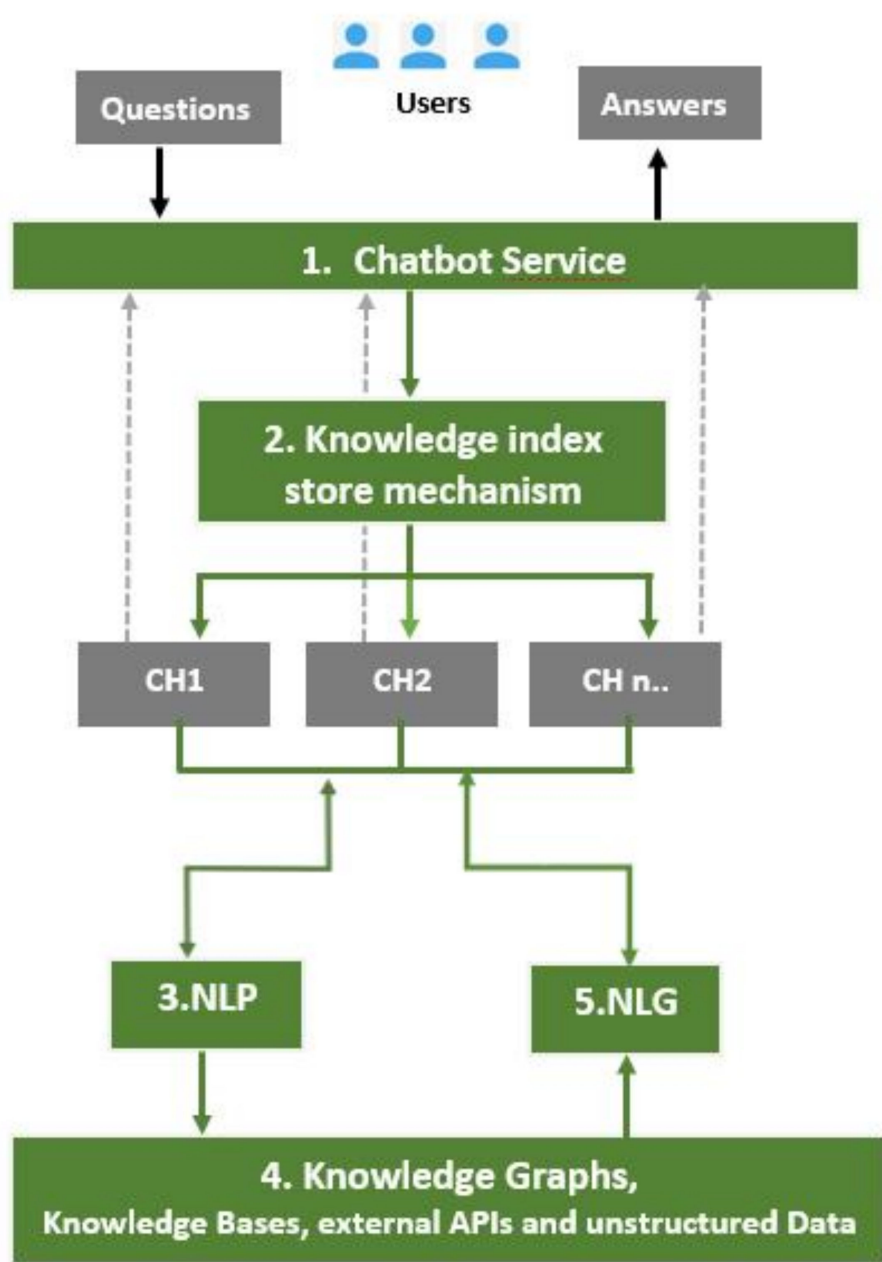

Figure 2. The proposed five-components multi-chatbot collaborative architecture.

The aim of the proposed architecture is to develop a conversational AI multi-chatbot distributed system that can deploy human-like conversations such as the following example (taken from a scenario in Nikos Kazantzakis (NK) museum in Greece):

- Visitor: What is presented in this museum hall?

- Infobot (Eleni): You are at the hall that the life of Nikos Kazantzakis is presented. Would you like to learn more about his life?

- Visitor: Yes, that would be great!

- Infobot (Eleni): Sure, your collection guide will be with you soon. You may always return to our discussion and ask me general questions about the museum.

- "NK Life" Collection Chatbot (George): Hello, I am the collection guide of Nikos Kazantzakis life. He was born in Heraklion. His father originated from the village that his museum is built, Myrtia. Would you like to tell you more or you prefer to see his life through an artefact journey in the museum?

- Visitor: Let's start the journey!

- "NK Life" Collection Chatbot (George): I believe that you are now in front of the first artifact showcase, where Kazantzakis' Early Life Memorabilia are presented.

- Visitor: Yes, I can see them. They look so interesting. But who are these women on the picture on the left?

- "NK Life" Collection Chatbot (George): Oh, what a lovely picture! These are his mother and his beloved sisters Anastasia and Eleni. Their dresses are so beautiful. And look at the left is also his mother's ring! Would like to hear the story of this ring?

- Visitor: No, I would like to know about his first book! 
- "NK Life" Collection Chatbot (George): His first book was a young novel about love, life and death. He was in Athens, studying law and was so in love with a girl. If you want to learn more about this book or others we should ask it from another guide, my colleague John (Books Collection Chabot).

- Visitor: no, it is fine, please tell me the story about when his was a law student.

- "NK Life" Collection Chatbot (George): On the upper right corner of the showcase, you can see a picture of him as a first-year undergraduate at the University of Athens. He studied law ....

- Visitor: And where can I buy a book about his life?

- "NK Life" Collection Chatbot (George): For this information you will have to get your answer from my colleague Eleni (InfoBot). Please excuse me. You may always return to our discussion and ask me questions about NK life.

- Infobot (Eleni): Our bookstore is at the entrance of the museum and an online store is always available at our website.

A key objective of the proposed approach is to customize such a conversation in a way that it looks like discussing in first person either with the exhibit itself or the writer, guide, presenter of a certain thematic. Providing a personality trait to an AI chatbot delivers a more engaging experience for the visitors, as communication in the first person is always more effective [146]. This is suitable in cases where special purpose chatbots are integrated in the system, such as conversational avatars. For instance, discussing with an avatar looking such as the famous Nikos Kazantzakis writer, is more realistic when this is performed in the first person.

For demonstration purposes, Figure 3 depicts the interaction of visitors (human agents) and the distributed and collaborative system of three different chatbots (software agents). This choice has been made to point out the capacity of the proposed approach to setup the multi-chatbot system in a way that can lookup/search for the requested knowledge in a distributed manner, i.e., among the network of interconnected/interrelated museum thematic collections or exhibits. Such a network is built offline by the museum curators, as they are familiar with the 'stories' that each thematic collection/exhibit can narrate to the visitors. This interconnection/interrelation is formally put in the knowledge structure that each chatbot have for lookup/search during the QA processing. So, if a question cannot be answered by a thematic collection chatbot due to lack of knowledge (i.e., its KG is missing the related knowledge), the chatbot may lookup/search in other thematic collection chatbots' knowledge or in the LOD cloud for relative knowledge.

In addition, a conversational infobot (MuseumBot) can also collaborate with thematic KG-based chatbots (CollectionBots) and assist visitors by answering general questions about the museum or other informative topics such as the weather or the history of the museum's town, the cost of a book at museum's e-shop, etc. The MuseumBot can also take the role of the host of a conversation between all agents (human and software), though visitors are able to select the chatbot they believe is able to address their questions, and to determine their host chatbot. The role of the host chatbot can be shared among the chatbots based on optimization strategies and data analytics of conversations as well as on the profiling of visitors. The following entities 'live' in the example museum chatbot environment that follows our proposed architecture:

- Museum Chatbot Users (MCU): the visitors of the museum (human agents),

- MuseumBot (MB): the informative chatbot (infobot) software agent

- CollectionBot (CB): the thematic collections chatbot software agents 


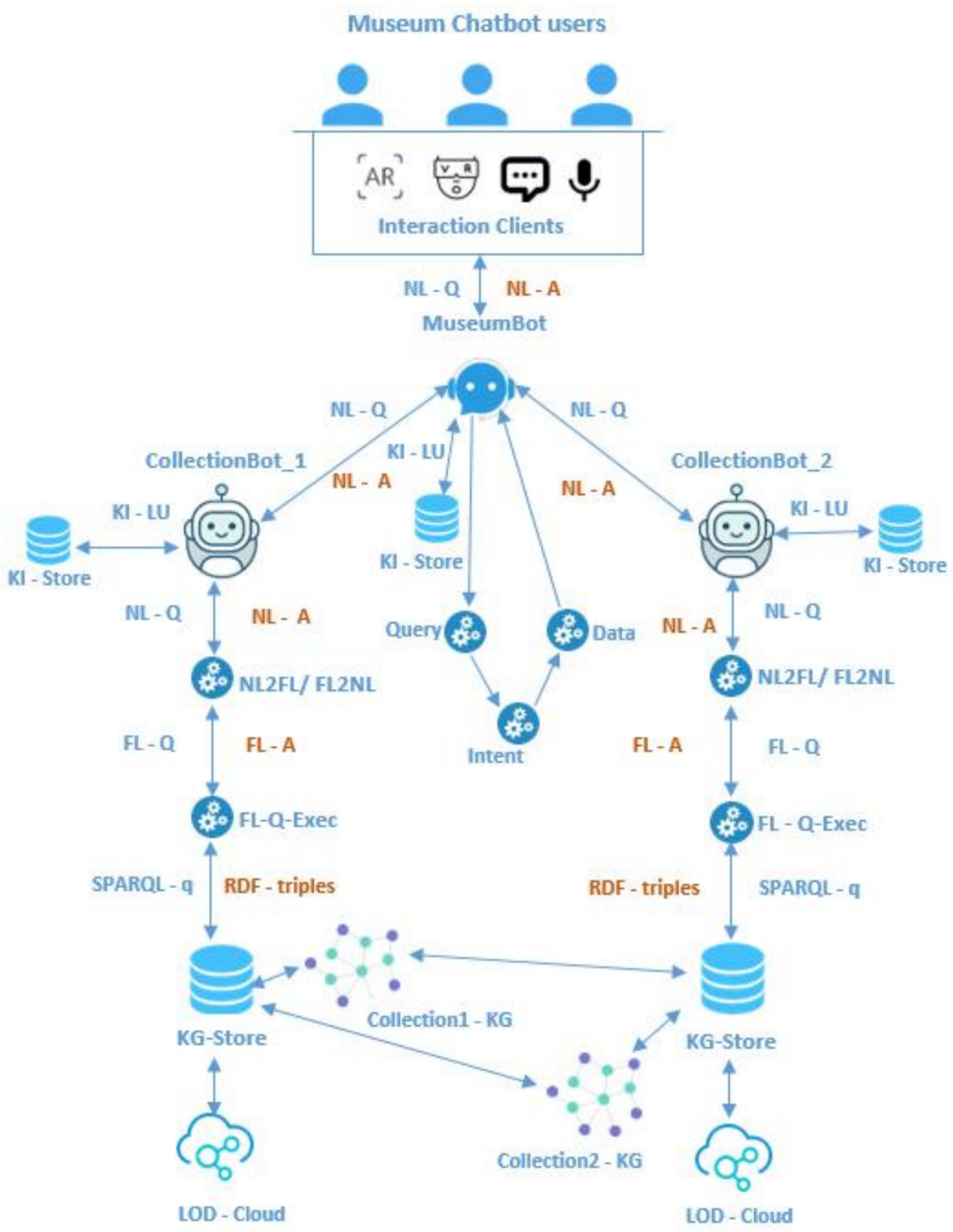

Figure 3. An example of a multi-chatbot graph-based architecture.

In terms of data, information and knowledge, the following are present in such an environment: (a) Knowledge Graph (KG), (b) Natural Language Question (NL-Q), (c) Natural Language Answer (NL-A), (d) Formal Language Question (FL-Q), (e) Formal Language Answer (FL-A), (f) SPARQL query (SPARQL-q), (g) RDF triples (RDF-triples). In terms of storage, the environment involves: (a) Knowledge Graphs in RDF triples store (KG-store), (b) a Knowledge Index store (KI-store), and (c) the Linked Open Data cloud (LOD-cloud). Finally, the processing of data, information and knowledge requires the following components: (a) Natural to Formal Language conversion (NL2FL), (b) Formal to Natural Language conversion (FL2NL), (c) Knowledge Index Lookup (KI-LU), (d) Formal Language Question Execution (FL-Q-Exec).

In Figure 3 the example presents museum visitors interacting through a chatbot dialog manager with two KG-based CBs and one MB. In this scenario the MB is acting as the host of the discussion. An example flow of the scenario is the following:

1. Visitors interact with the host chatbot (MB) (via Text, Voice, AR, or VR) to reach the CollectionBot of their interest (CB1 or CB2). 
2. $\mathrm{MB}$ searches the KI-stores using KI-LU, discovers the stored knowledge about the thematic collections and selects which $\mathrm{CB}$ can provide the appropriate answer.

3. If the KI-store recognizes the intent of a visitors NL-Q, initiates the chosen CB knowledge extraction process. $\mathrm{CB}$, with its NL2FL component, translates the NL-Q of the visitors to formal syntax and sends it to the FL-Q-Exec. At the FL-Q-Exec, FL-Q are turned into SPARQL-q in order to be looked-up to KG-store and retrieve the right answer. KGs are stored at the KG-store (as RDF triples). If an answer is not found in the matched KG-store, an additional search is conducted to the other KGs of the museum or to the connected LOD cloud sources.

4. When the matched RDF triples are retrieved, the FL-Q-Exec sends the FL-A to the FL2NL component.

5. In the FL2NL component, NLG techniques are used for the transformation of the FL-A to NL-A.

6. Finally, visitors receive the NL-As through their device user interface (Text, Voice, AR, VR).

At any point of the conversation visitors might place a NL-Q about knowledge related to the museum, e.g., the location of the museum's shop. A look-up task identifies the intent and sends the NL-Q to the museum chatbot and provides the proper NL-A.

Summarizing, the proposed KG-based, distributed, and collaborative multi-chatbot conversational AI system architecture is focusing in providing answers to the main challenges that chatbot applications face:

(a) The integration of KGs contribution in rich structured machine-understandable knowledge to the museum chatbots, solving the problem of limited and unstructured knowledge sources.

(b) The distributed and collaborative approach contribution in the capability of answering more (if not all) questions, and at the same time delivers a system that can be easily updated and expanded (with new collaborative chatbots).

\section{Validating the Approach at Nikos Kazantzakis Museum}

The recently renovated Museum of Nikos Kazantzakis is located in the historical village of Myrtia or Varvaroi, just $15 \mathrm{~km}$ from Heraklion town. The Nikos Kazantzakis Museum pays tribute to the important intellectual, author, thinker, philosopher, politician, and traveler Nikos Kazantzakis. The Museum was founded by set and costume designer Yiorgos Anemoyiannis, a pioneering figure in Greek theatre. The Museum holds manuscripts and notes by the author; samples of his correspondence with major thinkers, politicians, and authors of his time; first editions of his works in Greek and other languages; rare photographic material; souvenirs from his travels; personal effects; models, costumes and other material from theatre productions of his works in Greece and abroad; portraits and sculptures of the author; dozens of foreign-language editions of his works from all over the world. The Museum has an active webpage at https:/ / www.kazantzaki.gr/en (retrieved on 30 August 2021) which presents the work of Nikos Kazantzakis through its exhibitions. Also contains an e-shop and an online virtual tour [147].

The design and implementation of an AI chatbot application for Nikos Kazantzakis Museum (MNK) is under a funded EU regional program. The application will provide a guided tour to the visitors of the museum that we will be assisted by chatbot agents. As MNK is a small museum, the designed chatbot application must provide an interesting tour that will cover all aspects of the life and the work of Nikos Kazantzakis. Certain exhibits will be selected in order to be presented through the application. The designed system must be simple, easy in use and must not disrupt visitors from their physical visit but only to assist and enrich their knowledge. Furthermore, the designed system will be open source, expandable and easily updated through a web system. The designed chatbots must be informative, conversational, and friendly in a way that visitors will engage and conduct an interesting conversation. The use of the voice of Nikos Kazantzakis as the narrator of 
the guide-chatbot and the use of fully conversational chatbot agent are two desirables and rather challenging aspects.

\section{Conclusions}

The aim of this paper is to survey existing conversational AI technology and related applications, and to propose a novel approach for the design of a multi-chatbot system that utilizes KGs, has strong conversational skills by exploiting both NLU and ML techniques, and rely on the distributed and collaborative aspects of AI. The proposed KG-based, distributed, and collaborative multi-chatbot conversational AI system architecture is focusing in providing an answer to the main challenges that chatbot applications face. KGs contribute rich structured machine-understandable knowledge to the museum chatbots, solving the problem of limited and unstructured knowledge sources. The distributed and collaborative approach contributes the capability of answering more (if not all) questions, and at the same time delivers a system that can be easily updated and expanded (with new collaborative chatbots).

Several technical challenges such as intent routing, turn taking, context switching, server handling and other technical issues arise in the proposed multi-chatbot architecture. Developers are proposing the use of an orchestration, or host or mega-agent bot that is orchestrating the chatbots and (re-)routes the users' intents. However, this solution is by default excluding the real collaboration capability from a multi-chatbot system. In addition, ethical or legal issues such as biased training, users' misbehavior, content violations, etc., arise, leading chatbot developers, museum curators and users to question the need of fully human-like chatbots.

Our future work concerns the validation of the proposed system architecture by developing a KG-based, distributed, and collaborative multi-chatbot conversational AI system for Nikos Kazantzakis Museum in Greece and examine all the challenges that arise. The multi-chatbot system has been already designed and initial experiments of basic components have been developed using Rasa Framework and a KG in Neo4J graph database. Our plans also include the evaluation of automated knowledge extraction techniques that will feed the KGs of a multi-chatbot system with new knowledge from various Web sources. It is important to stress that the success of the proposed approach is depended on the quality of the KGs that will be integrated as the underlying knowledge representation technology. Thus, we will ensure that any representational problems will be solved conceptually on a formal level before an implementation attempt in the sense of proof-of-concept implementation is performed.

Author Contributions: Conceptualization, K.K. and S.V.; methodology, S.V.; software, S.V. and G.K.; validation, S.V., G.K. and D.P.; formal analysis, S.V.; investigation, K.K. and S.V.; resources, S.V. and D.P.; writing—original draft preparation, S.V.; writing—review and editing, K.K.; visualization, S.V.; supervision, K.K.; project administration, S.V.; funding acquisition, S.V. All authors have read and agreed to the published version of the manuscript.

Funding: This research was funded by the following two projects: "MEMOBOT, talking with Kazantzakis: Intelligent Mixed Reality Experiences", which is part of the single RTDI State Aid Action "RESEARCH-CREATE-INNOVATE" funded by the Greek Operational Programme Competitiveness, Entrepreneurship and Innovation 2014-2020 (EPAnEK). Project number: T2E $\Delta$ K-03334. “MuBot, a smart guide for museums", which is part of Action 1.b.3 "Research and development by SMEs in the Sector of RIS3Crete" funded by the European Fund of Regional Development (ERDF) in the framework of the Operational Program CRETE 2014-2020 of the NSRF 2014-2020. Project number: KPHP3-0026737.

Institutional Review Board Statement: Not applicable.

Informed Consent Statement: Not applicable.

Acknowledgments: We would like to thank Ladikou Stamatia as well as the staff of Nikos Kazantzakis Museum for their valuable input. 
Conflicts of Interest: The authors declare no conflict of interest.

\section{References}

1. Ram, A.; Prasad, R.; Khatri, C.; Venkatesh, A.; Gabriel, R.; Liu, Q. Conversational Ai: The Science behind the Alexa Prize. arXiv 2018, arXiv:1801.03604.

2. Levesque, H.J. Common Sense, the Turing Test, and the Quest for Real AI: Reflections on Natural and Artificial Intelligence, 1st ed.; MIT Press: London, UK, 2017.

3. Achladianakis, A. The Chatbots and the Possibilities Provided on Marketing. Clearing and Inclusion of Indicative Chatbot in a Content Management System (CMS). Master's Thesis, T.E.I. of Crete, Agios Nikolaos, Greece, 29 October 2019.

4. Vassos, S.; Malliaraki, E.; Falco, F.D.; Di Maggio, J.; Massimetti, M.; Nocentini, M.G.; Testa, A. Art-Bots: Toward Chat-Based Conversational Experiences in Museums. In Proceedings of the 9th International Conference on Interactive Digital Storytelling, ICIDS 2016, Los Angeles, CA, USA, 15-18 November 2016; pp. 433-437. [CrossRef]

5. Boiano, S.; Borda, A.; Gaia, G.; Rossi, S.; Cuomo, P. Chatbots and New Audience Opportunities for Museums and Heritage Organisations. In Proceedings of the Electronic Visualisation and the Arts (EVA 2018), London, UK, 9-13 July 2018 ; pp. 164-171. [CrossRef]

6. Tzouganatou, A. Can Heritage Bots Thrive? Toward Future Engagement in Cultural Heritage. Adv. Archaeol. Pract. 2018, 6, 377-383. [CrossRef]

7. Schaffer, S.; Gustke, O.; Oldemeier, J.; Reithinger, N. Towards chatbots in the museum. CEUR Workshop Proc. $2018,2176,1-7$.

8. Roussou, M.; Perry, S.; Katifori, A.; Vassos, S.; Tzouganatou, A.; McKinney, S. Transformation through Provocation? In Proceedings of the 2019 CHI Conference on Human Factors in Computing Systems, CHI, Glasgow, UK, 4-9 May 2019. [CrossRef]

9. Gaia, G.; Boiano, S.; Borda, A. Engaging Museum Visitors with AI: The Case of Chatbots. In Museums and Digital Culture; Giannini, T., Bowen, J., Eds.; Springer Series on Cultural Computing: Berlin/Heidelberg, Germany, 2019; pp. 309-329. [CrossRef]

10. Ławrynowicz, A.; Tresp, V. Introducing machine learning. In Perspectives on Ontology Learning; Lehmann, J., Voelker, J., Eds.; AKA Heidelberg/IOS Press: Heidelberg, Germany; pp. 35-50.

11. Suta, P.; Lan, X.; Wu, B.; Mongkolnam, P.; Chan, J.H. An Overview of Machine Learning in Chatbots. Int. J. Mech. Eng. Robot. Res. 2020, 9, 502-510. [CrossRef]

12. Navigli, R. Natural Language Understanding: Instructions for (Present and Future) Use. IJCAI Int. Jt. Conf. Artif. Intell. 2018, 5697-5702. [CrossRef]

13. Nadkarni, P.M.; Ohno-Machado, L.; Chapman, W.W. Natural language processing: An introduction. JAMIA 2011, 18, 5-544. [CrossRef]

14. Yan, J.; Wang, C.; Cheng, W.; Gao, M.; Zhou, A. A retrospective of knowledge graphs. Front. Comput. Sci. 2016, 12, 55-74. [CrossRef]

15. Bonatti, P.A.; Decker, S.; Polleres, A.; Presutti, V. Knowledge graphs: New directions for knowledge representation on the semantic web. Rep. Dagstuhl Semin. 2019, 8, 29-111. [CrossRef]

16. Varitimiadis, S.; Kotis, K.; Spiliotopoulos, D.; Vassilakis, C.; Margaris, D. “Talking” Triples to Museum Chatbots. Int. Conf. Hum. Comput. Interact. HCI 2020, 35, 281-299.

17. Academia.edu—Share Research. Available online: https://www.academia.edu/ (accessed on 8 August 2021).

18. ResearchGate-Find and Share Research. Available online: https://www.researchgate.net/ (accessed on 8 August 2021).

19. Google. Available online: https:/ /www.google.com/ (accessed on 8 August 2021).

20. Semantic Scholar-AI-Powered Research Tool. Available online: https://www.semanticscholar.org/ (accessed on 8 August 2021).

21. Association for Computing Machinery. Available online: https://www.acm.org/ (accessed on 8 August 2021).

22. IEEE, Advancing Technology for Humanity. Available online: https://www.ieee.org/ (accessed on 8 August 2021).

23. Springer-International Publisher Science and Technology. Available online: https://www.springer.com/gp (accessed on 8 August 2021).

24. Kölbl, A.; Rosen, M. Talk to Me! Chatbots in Museums, A Chronological Overview. Available online: https://zkm.de/en/talk-tome-chatbots-in-museums (accessed on 17 March 2021).

25. Charr, M. How Are Museums Using Chatbots? Available online: https://www.museumnext.com/article/how-are-museumsare-using-chatbots / (accessed on 17 March 2021).

26. Allen, J.F.; Byron, D.K.; Dzikovska, M.; Ferguson, G.; Galescu, L.; Stent, A. Toward conversational human-computer interaction. AI Mag. 2001, 22, 2738.

27. Følstad, A.; Brandtzaeg, P.B. Chatbots and the new world of HCI. Interactions 2017, 24, 38-42. [CrossRef]

28. Valtolina, S.; Barricelli, B.R.; Gaetano, S.D.; Diliberto, P. Chatbots and conversational interfaces: Three domains of use. CEUR Workshop Proc. 2018, 2101, 62-70.

29. Kotis, K.I.; Zachila, K.; Paparidis, E. Machine Learning Meets the Semantic Web. Artif. Intell. Adv. 2021, 3, 1. [CrossRef]

30. Wehle, H. Machine Learning, Deep Learning and AI: What's the Difference? Conference: Data Scientist Innovation Day. 2017. Available online: https://www.researchgate.net/publication/318900216_Machine_Learning_Deep_Learning_and_AI_What T1 \textquoterights_the_Difference (accessed on 30 August 2021).

31. Rivero, A. Next Generation of Chatbots with NLP Services and Graphs in Chatbots Life. 2018. Available online: https: //chatbotslife.com/next-generation-of-chatbots-with-nlp-services-and-graphs-cd811a8165d7 (accessed on 10 March 2021). 
32. Orth, A. Building Chatbots with Dialogflow and Grank.AI in Grank.ai. 2017. Available online: https://blog.grakn.ai/chatbotsand-grakn-ai-67563c64cfde (accessed on 10 March 2021).

33. Cambria, E.; White, B. Jumping NLP Curves: A Review of Natural Language Processing Research. IEEE Comput. Intell. Mag. 2014, 9, 48-57. [CrossRef]

34. Staykova, K. Natural Language Generation and Semantic Technologies. Cybern. Inf. Technol. 2014, 14, 3-23. [CrossRef]

35. Riedl, M.O.; Bulitko, V. Interactive Narrative: An Intelligent Systems Approach. AI Mag. 2012, 34, 67. [CrossRef]

36. Resource Description Framework (RDF). Available online: http://www.w3.org/RDF/ (accessed on 28 August 2012).

37. SPARQL Query Language for RDF. Available online: http:/ / www.w3.org/TR/rdf-sparql-query/ (accessed on 4 April 2011).

38. World Wide Web Consortium (W3C) OWL Working Group. Web Ontology Language (OWL). 2012. Available online: https: / / www.w3.org/OWL/ (accessed on 21 July 2019).

39. Schuetzler, R.M.; Giboney, J.S.; Grimes, G.M.; Nunamaker, J.F. The influence of conversational agent embodiment and conversational relevance on socially desirable responding. Decis. Support Syst. 2018, 114, 94-102. [CrossRef]

40. Van Pinxteren, M.M.; Pluymaekers, M.; Lemmink, J.G. Human-Like communication in conversational agents: A literature review and research agenda. J. Serv. Manag. 2020, 31, 203-225. [CrossRef]

41. Svenningsson, N.; Faraon, M. Artificial Intelligence in Conversational Agents: A Study of Factors Related to Perceived Humanness in Chatbots. In Proceedings of the AICCC 2019 2nd Artificial Intelligence and Cloud Computing Conference, Kobe, Japan, 21-23 December 2019; pp. 151-161. [CrossRef]

42. Donkelaar, L. How Human Should a Chatbot Be? Master's Thesis, University of Twente, Twente, Netherlands, 6 November 2018.

43. Wikipedia, Distributed Collaboration. Available online: https://en.wikipedia.org/wiki/Distributed_collaboration (accessed on 8 August 2021).

44. Martin, C.; Schreckenghost, D.; Bonasso, P.; Kortenkamp, D.; Milam, T.; Thronesbery, C. An environment for distributed collaboration among humans and software agents. In Proceedings of the AAMAS'03-Second International Joint Conference on Autonomous Agents and Multiagent Systems 2003, Melbourne, Australia, 14-18 July 2003; pp. 1062-1063. [CrossRef]

45. Han, S.-H. A Study on the Distributed Collaborative Architectural Design System. J. Asian Arch. Build. Eng. 2006, 5, 67-74. [CrossRef]

46. Bosse, S. Distributed Serverless Chat Bot Networks using Mobile Agents: A Distributed Data Base Model for Social Networking and Data Analytics. In Proceedings of the ICAART 2021-13th International Conference on Agents and Artificial Intelligence 2021, Virtual, 4-6 February 2021; pp. 398-405. [CrossRef]

47. Subramaniam, S.; Aggarwal, P.; Dasgupta, G.B.; Paradkar, A. COBOTS-A Cognitive Multi-Bot Conversational Framework for Technical Support. In Proceedings of the International Joint Conference on Autonomous Agents and Multiagent Systems, AAMAS, Stockholm, Sweden, 10-15 July 2018; pp. 597-604.

48. OpenDialog Manifesto. Available online: https:/ / opendialog.ai/manifesto/ (accessed on 8 August 2021).

49. Drissner, A. Rasa, Meta Bots: Why They're Probably Not the Solution to Your Problems. Available online: https://www.youtube. com/watch?v=BwIj5C-r5ZE (accessed on 8 August 2021).

50. Drissner, A. Rasa, Many Bots, One Voice: Seamless Multi-Bot Orchestration in the Enterprise I L3-AI 2021. Available online: https: / / www.youtube.com/watch?v=m5z8ITIO3Vk (accessed on 8 August 2021).

51. Kore.ai. Universal Virtual Assistants: Orchestrate Tasks from Multiple Specialized Virtual Assistants. Available online: https: // kore.ai/platform/features/universal-bots/ (accessed on 8 August 2021).

52. Servisbot, Scale Your Bots Exponentially with Multi-Model NLP Orchestration. Available online: https://servisbot.com/multimodel-nlp-orchestration-on-rasa/ (accessed on 8 August 2021).

53. Chaves, A.P.; Gerosa, M.A. Single or Multiple Conversational Agents? An Interactional Coherence Comparison. In Proceedings of the Conference on Human Factors in Computing Systems 2018, Montreal, Canada, 21-26 April 2018; Volume 191, pp. 1-13. [CrossRef]

54. Pinhanez, C.S.; Candello, H.; Pichiliani, M.C.; Vasconcelos, M.; Guerra, M.; de Bayser, M.G.; Cavalin, P. Different but Equal: Comparing User Collaboration with Digital Personal Assistants vs. Teams of Expert Agents. arXiv 2018, arXiv:1808.08157.

55. Chatfuel Directory of Chatbots: National Art Museum of the Republic of Belarus. Available online: https://chatfuel.com/bot/ The-National-Art-Museum-of-the-Republic-of-Belarus-1904070043163955 (accessed on 17 March 2021).

56. Boiano, S.; Borda, A.; Cuomo, P.; Gaia, G.; Rossi, S. Chatbots in Museums: Hype or Opportunity? MuseWeb. Available online: https://www.museweb.net/chatbots-in-museums-hype-or-opportunity-\%E2\%80\%A8/ (accessed on 17 March 2021).

57. Netomi: AI Customer Service: Customer Support Chatbot Solutions. Available online: https://www.netomi.com/ (accessed on 17 March 2021).

58. Museo di arte Moderna e Contemporanea di Trento e Rovereto, THE MART CHAT. Available online: http://www.mart.tn.it/ martmuseumbot-en (accessed on 17 March 2021).

59. Engineering. Chatbot, AI and Gamification for the MAXXI Museum in Rome. Available online: https://www.eng.it/en/casestudies / chatbot-museo-maxxi (accessed on 17 March 2021).

60. Pequignot, C. Teaching a Titanosaur to Talk: Conversational UX Design for Field Museum. Available online: https:// purplerockscissors.com/blog/teaching-a-titanosaur-to-talk (accessed on 17 March 2021).

61. Field Museum. If the World's Biggest Dinosaur Could Talk, What Would He Say? Available online: https://www.fieldmuseum. org/exhibitions/webby-entry-message-maximo (accessed on 17 March 2021). 
62. Antheon. Van Gogh Museum Automates Answers to Frequently Asked Questions with Microsoft Chatbot. Available online: https:/ / www.antheon.nl/projects/van-gogh-museum-chatbot/ (accessed on 17 March 2021).

63. Github Repository, Andybot-Botpress. Available online: https://github.com/CMP-Studio/andybot-botpress (accessed on 17 March 2021).

64. Ticknor, S. A Chatbot Adventure. Available online: https://studio.carnegiemuseums.org/a-chatbot-adventure-c729812571ea (accessed on 17 March 2021).

65. Meet CarnegieBot at AAM! Available online: https://studio.carnegiemuseums.org/meet-carnegiebot-at-aam-d3cef28720aa (accessed on 17 March 2021).

66. Morena, D. IRIS+Part One: Designing + Coding a Museum AI. Available online: https://www.aam-us.org/2018/06/12/irispart-one-designing-coding-a-museum-ai/ (accessed on 17 March 2021).

67. Renoldi, G. IRIS+ I Uma nova experiência no Museu do Amanhã. Available online: https://museudoamanha.org.br/pt-br/ irismais (accessed on 17 March 2021).

68. IRIS+Uma nova Experiência no Museu do Amanhã. 2017. Available online: https://www.youtube.com/watch?v=os_shHyK3-0 (accessed on 17 March 2021).

69. Iris Screen Capture 2A1. 2018. Available online: https:/ / www.youtube.com/watch?v=0944e3x-hq4 (accessed on 17 March 2021).

70. MAMBA (Museo de Arte Moderno de Buenos Aires)—Dialogue with the Artwork. Available online: http://www. brunobarbosaworks.com/mamba (accessed on 17 March 2021).

71. IBM Press Release. IBM and Pinacoteca de São Paulo Train IBM Watson to Talk with Visitors about Works of Art. Available online: https:/ / www-03.ibm.com/press/us/en/pressrelease/52443.wss (accessed on 17 March 2021).

72. Richardson, J. Giving Art a Voice with an Artificial Intelligence Audio Guide. Available online: https://www.museumnext.com/ article/artificial-intelligence-audio-guide/ (accessed on 17 March 2021).

73. Ask Mona Studio Webpage. Available online: https:/ / studio.askmona.fr/en (accessed on 17 March 2021).

74. Museums of the city of Paris. Chatbot: Paris Musées launches a Conversational Interface to Direct Visitors. Available online: https:/ / www.parismusees.paris.fr/en/actualite/chatbot-paris-musees-launches-a-conversational-interface-to-directvisitors (accessed on 17 March 2021).

75. Présentation Ask Mona Studio. Available online: https:/ /www.youtube.com/watch?v=9jDCaqw-T2Y (accessed on 17 March 2021).

76. Ask Sarah FB Messenger Bot. Available online: https://www.messenger.com/t/273861966942/ (accessed on 17 March 2021).

77. Multilingual Europeana Chatbot Project. Available online: https:/ / culturebot.eu/ (accessed on 17 March 2021).

78. Jewish Heritage Network, Museum Chatbot-State-of-The-Art Machine Learning Tools to Build Contextual Chatbots and Assistants That Engage Visitors with Cultural Content. Available online: https://jhn.ngo/services/chatbot (accessed on 17 March 2021).

79. POLIN Museum, The Culture Chatbot: A New Facebook Communication Tool. Available online: https://www.polin.pl/en/ culture-chatbot (accessed on 17 March 2021).

80. ICCU Culture Chatbot. Available online: https://www.iccu.sbn.it/en/activities/international-activities/culture-chatbot/ (accessed on 17 March 2021).

81. Rasa Open Source Conversational AI. Culture Chatbot: A Virtual Museum Tour Guide. Available online: https:/ rasa.com/ showcase/jhn-ams / (accessed on 17 March 2021).

82. Kats, P.; Evans, G. The Culture Chatbot-Engaging Visitors with Your Collections. Available online: https://pro.europeana.eu/ post/the-culture-chatbot-engaging-visitors-with-your-collections (accessed on 17 March 2021).

83. Fondation Cartier-Chatbot Context Demonstration. Available online: https://vimeo.com/253466487 (accessed on 17 March 2021).

84. Steven, R. Building a Chatbot for Fondation Cartier. Available online: https://www.creativereview.co.uk/building-a-chatbot-forfondation-cartier-paris / (accessed on 17 March 2021).

85. Gerber, K. Tour Akron Art Museum with Dot the Chatbot. Available online: https://www.theformgroup.com/articles/2018/10/ 17/tour-akron-art-museum-with-dot-the-chatbot (accessed on 17 March 2021).

86. Litt, S. Dot, the New Akron Art Museum Chatbot, Wants to Get You Talking About Art and Life. Available online: https: / /www.cleveland.com/arts/2018/08/dot_the_new_akron_art_museum_c.html (accessed on 17 March 2021).

87. Varitimiadis, S.; Kotis, K.; Skamagis, A.; Tzortzakakis, A.; Tsekouras, G.; Spiliotopoulos, D. Towards implementing an AI chatbot platform for museums. In Proceedings of the 2nd International Conference on Cultural Informatics, Communication \& Media Studies 2019, Mytilene, Greece, 13-15 June 2019. [CrossRef]

88. Styx, L. How Are Museums Using Artificial Intelligence and Is AI the Future of Museums? Available online: https://www. museumnext.com/article/artificial-intelligence-and-the-future-of-museums/ (accessed on 17 March 2021).

89. Radziwill, N.M.; Benton, M.C. Evaluating Quality of Chatbots and Intelligent Conversational Agents. arXiv 2017, arXiv:1704.04579.

90. Abran, A.; Khelifi, A.; Suryn, W.; Seffah, A. Consolidating the ISO usability models. In Proceedings of the 11th International Software Quality Management Conference 2003, Glasgow, UK, 23-25 April 2003; Volume 2003, pp. $23-25$.

91. Saaty, R. The Analytic Hierarchy Process-What It Is and How It Is Used. Math. Model. 1987, 9, 161-176. [CrossRef] 
92. Kostelník, P.; Pisařovic, I.; Muroň, M.; Dařena, F.; Procházka, D. Chatbots for Enterprises: Outlook. Acta Univ. Agric. Silvic. Mendel. Brun. 2019, 67, 1541-1550. [CrossRef]

93. Salmeron, J.L.; Herrero, I. An AHP-Based methodology to rank critical success factors of executive information systems. Comput. Stand. Interfaces 2005, 28, 1-12. [CrossRef]

94. Vaidya, O.S.; Kumar, S. Analytic hierarchy process: An overview of applications. Eur. J. Oper. Res. 2006, 169, 1-29. [CrossRef]

95. Couto, J. Building A Chatbot: Analysis and Limitations of Modern Platforms-DZone AI. Available online: https://dzone.com/ articles/building-a-chatbot-analysis-amp-limitations-of-mod (accessed on 17 March 2021).

96. Chatfuel, Chatbot Platform for Facebook Messenger. Available online: https:/ / chatfuel.com (accessed on 17 March 2021).

97. Octane.ai, Commercial and Social Media Bots. Available online: https:/ / octaneai.com/ (accessed on 17 March 2021).

98. Motion.ai, A visual Chatbot Builder. Available online: https:/ /www.motion.ai/ (accessed on 17 March 2021).

99. PandoraBots, Intelligent Conversational Agents. Available online: https://home.pandorabots.com/home.html (accessed on 17 March 2021).

100. Dialogflow, Build Natural and Rich Conversational Experiences. Available online: https://cloud.google.com/dialogflow (accessed on 17 March 2021).

101. Wit.ai. Natural Language for Developers. Available online: https:/ / wit.ai/ (accessed on 17 March 2021).

102. Watson Assistant, More than A Chatbot. Available online: https://www.ibm.com/cloud/watson-assistant/ (accessed on 17 March 2021).

103. Microsoft Bot Framework. A Comprehensive Framework for Building Enterprise-Grade Conversational AI Experiences. Available online: https: / / dev.botframework.com/ (accessed on 4 May 2021).

104. Amazon Lex. Conversational Interfaces for Your Applications. Available online: https://aws.amazon.com/lex/ (accessed on 17 March 2021).

105. Rasa. Open Source Conversational AI. Available online: https:/ / rasa.com/ (accessed on 17 March 2021).

106. Botpress-Open Source Conversational AI Platform. Available online: https:/ /botpress.com/ (accessed on 17 March 2021).

107. Opendialog-Open Source Enterprise Conversational, AI. Available online: https:/ /www.opendialog.ai/ (accessed on 4 May 2021).

108. Ujwalakavya, J.; Deepamala, N. Chatbot for Business Environment Using Botpress Conversational AI Platform. Int. Res. J. Eng. Technol. (IRJET) 2020, 7, 6503-6507.

109. Dgraph, A Native GraphQL Graph Database. Available online: https:/ / dgraph.io/ (accessed on 10 May 2021).

110. Grakn, An Intelligent Database-A Knowledge Graph. Available online: https:/ / grakn.ai/ (accessed on 10 May 2021).

111. Neo4j's A Highly Scalable Native Graph Database. Available online: https://neo4j.com/ (accessed on 10 May 2021 ).

112. OrientDB - The First Multi-Model Open Source NoSQL DBMS. Available online: https:/ / orientdb.org/ (accessed on 10 May 2021).

113. GraphDB, An Enterprise Ready Semantic Graph Database. Available online: https://graphdb.ontotext.com/ (accessed on 10 May 2021).

114. Boné, J.; Ferreira, J.C.; Ribeiro, R.; Cadete, G. DisBot: A Portuguese Disaster Support Dynamic Knowledge Chatbot. Appl. Sci. 2020, 10, 9082. [CrossRef]

115. Braun, D.; Matthes, F. Towards a Framework for Classifying Chatbots. In Proceedings of the ICEIS 2019-21st International Conference on Enterprise Information Systems 2019, Heraklion, Greece, 3-5 May 2019; pp. 484-489. [CrossRef]

116. Shabbir, J.; Arshad, M.U.; Shahzad, W. NUBOT: Embedded Knowledge Graph with RASA Framework for Generating Semantic Intents Responses in Roman Urdu. arXiv 2021, arXiv:2102.10410.

117. Perez-Soler, S.; Juarez-Puerta, S.; Guerra, E.; de Lara, J. Choosing a Chatbot Development Tool. IEEE Softw. 2021, 38, 94-103. [CrossRef]

118. Braun, D.; Hernandez-Mendez, A.; Matthes, F.; Langen, M. Evaluating Natural Language Understanding Services for Conversational Question Answering Systems. In Proceedings of the SIGDIAL 2017-18th Annual Meeting of the Special Interest Group on Discourse and Dialogue 2017, Saarbrücken, Germany, 15-17 August 2017; pp. 174-185. [CrossRef]

119. Botfront, Enterprise-Grade Open Source Platform for Rasa Teams. Available online: https:/ /botfront.io/ (accessed on 4 May 2021).

120. Snatchbot, Principles of Bot Design. Available online: https://support.snatchbot.me/docs/principles-of-bot-design (accessed on 8 August 2021).

121. Ajay Inamdar, V. Development of College Enquiry Chatbot Using Snatchbot. Int. Res. J. Eng. Technol. 2019, 6, $1615-1618$.

122. Snatchbot, Create Smart Chatbots for Multi-Channel Messaging. Available online: https://snatchbot.me/ (accessed on 5 May 2021).

123. Patil, A.; Marimuthu, K.; Niranchana, R. Comparative study of cloud platforms to develop a Chatbot. Int. J. Eng. Technol. 2017, 6, 57-61. [CrossRef]

124. Kore.ai Virtual Assistant Platform. Available online: https:/ / kore.ai/ (accessed on 5 May 2021).

125. Kore.ai's Knowledge Graph Generator. Available online: https://github.com/Koredotcom/KnowledgeGraphGenerator\#readme (accessed on 5 May 2021).

126. DialogFlow, Mega Agents. Available online: https:/ / cloud.google.com/dialogflow/es/docs/agents-mega (accessed on 8 August 2021). 
127. Nimavat, K.; Champaneria, T. Chatbots: An Overview Types, Architecture, Tools and Future Possibilities. Int. J. Sci. Res. Dev. 2017, 5, 1019-1026.

128. Yoo, S.Y.; Jeong, O.R. Auto-Growing knowledge graph-based intelligent chatbot using BERT. ICIC Express Lett. 2020, 14, 67-73. [CrossRef]

129. IBM. Create Domain-Specific Bots Using an AGENT bot. Available online: https://developer.ibm.com/technologies/artificialintelligence/patterns/compose-bots-using-an-agent-bot/ (accessed on 8 August 2021).

130. Quamar, A.; Lei, C.; Miller, D.; Ozcan, F.; Kreulen, J.; Moore, R.J.; Efthymiou, V. An Ontology-Based Conversation System for Knowledge Bases. In Proceedings of the ACM SIGMOD International Conference on Management of Data 2020, Portland, OR, USA, 14-19 June 2020; pp. 361-376. [CrossRef]

131. Amato, F.; Marrone, S.; Moscato, V.; Piantadosi, G.; Picariello, A.; Sansone, C. Chatbots meet e-health: Automatizing healthcare. Farm. Zh. 2018, 3, 89-91.

132. Handoyo, E.; Arfan, M.; Soetrisno, Y.A.A.; Somantri, M.; Sofwan, A.; Sinuraya, E.W. Ticketing Chatbot Service using Serverless NLP Technology. In Proceedings of the 2018 th International Conference on Information Technology, Computer and Electrical Engineering, ICITACEE 2018, Semarang, Indonesia, 27-28 September 2018; pp. 325-330. [CrossRef]

133. Qaffas, A.A. Improvement of Chatbots Semantics Using Wit.ai and Word Sequence Kernel: Education Chatbot as a Case Study. Int. J. Mod. Educ. Comput. Sci. 2019, 11, 16-22. [CrossRef]

134. Vegesna, A.; Jain, P.; Porwal, D. Ontology based Chatbot (For E-Commerce Website). Int. J. Comput. Appl. 2018, 179, 51-55. [CrossRef]

135. Microsoft, Orchestrating Multiple Bots with Multilingual Support. Available online: https://devblogs.microsoft.com/cse/2017 /01/21/orchestrating-multiple-bots-with-multilingual-support/ (accessed on 8 August 2021).

136. Microsoft, Creating A Single Bot Service with Azure to Support Multiple Bot Applications. Available online: https://devblogs microsoft.com/cse/2017/01/10/creating-a-single-bot-service-to-support-multiple-bot-applications / (accessed on 8 August 2021).

137. Amazon Web Service, Integrating Amazon Connect and Amazon Lex with Third-party Systems. Available online: https: //aws.amazon.com/blogs/architecture/integrating-amazon-connect-and-amazon-lex-with-third-party-systems/ (accessed on 8 August 2021).

138. Kumar, S.; Rajagopal, P. Cloud Methodologies for A Seamless Integrated Chatbot. Int. J. Eng. Res. Technol. (IJERT) 2020, 9, 44-48.

139. Samuel, I.; Ogunkeye, F.A.; Olajube, A.; Awelewa, A. Development of a Voice Chatbot for Payment Using Amazon Lex Service with Eyowo as the Payment Platform. In Proceedings of the International Conference on Decision Aid Sciences and Application (DASA) 2020, Virtual, 8-9 November 2020; pp. 104-108. [CrossRef]

140. Goepel, K.D. Implementation of an Online Software Tool for the Analytic Hierarchy Process (AHP-OS). Int. J. Anal. Hierarchy Process. 2018, 10, 3. [CrossRef]

141. AHP Priority Calculator. Available online: https://bpmsg.com/ahp/ahp-calc.php (accessed on 10 May 2021).

142. Hallili, A. Toward An Ontology-Based Chatbot Endowed with Natural Language Processing and Generation. In Proceedings of the 26th European Summer School in Logic, Language \& Information, Tübingen, Germany, 11-22 August 2014.

143. Musen, M.A. The protégé project. AI Matters 2015, 1, 4-12. [CrossRef]

144. Al-Zubaide, H.; Issa, A.A. OntBot: Ontology based chatbot. In Proceedings of the International Symposium on Innovations in Information and Communications Technology 2011, Amman, Jordan, 29 November-1 December 2011; pp. 7-12. [CrossRef]

145. Ait-Mlouk, A.; Jiang, L. KBot: A Knowledge Graph Based ChatBot for Natural Language Understanding Over Linked Data. IEEE Access 2020, 8, 149220-149230. [CrossRef]

146. Zumstein, D.; Hundertmark, S. Chatbots: An interactive technology for personalized communication and transaction. Int. J. WWW/Internet 2018, 15, 96-109.

147. Nikos Kazantzakis Museum. Available online: https:/ /www.kazantzaki.gr/en (accessed on 17 March 2021). 